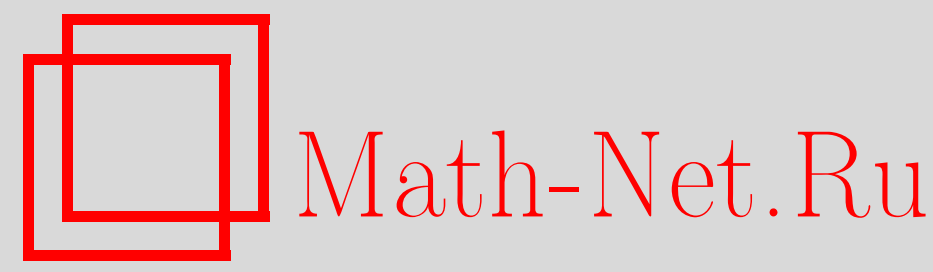

Л. И. Сазонов, Обоснование асимптотического разложения решения двумерной задачи обтекания при малых числах Рейнольдса, Изв. РАН. Сер. матем., 2003, том 67 , выпуск $5,125-154$

DOI: https://doi.org/10.4213/im454

Использование Общероссийского математического портала Math-Net.Ru подразумевает, что вы прочитали и согласны с пользовательским соглашением

http://www. mathnet.ru/rus/agreement

Параметры загрузки:

IP : 54.174 .149 .18

26 апреля 2023 г., 10:37:13

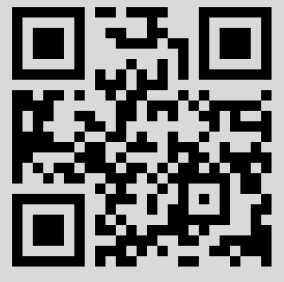


УДК 517.9

\author{
Л.И. Сазонов
}

\title{
Обоснование асимптотического разложения решения двумерной задачи обтекания при малых числах Рейнольдса
}

\footnotetext{
Строится и обосновывается полное асимптотическое разложение решения двумерной задачи обтекания ограниченного тела потоком вязкой жидкости при малых числах Рейнольдса.

Библиограбиия: 9 наименований.
}

\section{Введение}

Двумерная стационарная задача обтекания состоит в определении решения краевой задачи для системы Навье-Стокса

$$
\begin{gathered}
\Delta u-\nabla p=2 \varepsilon(u, \nabla) u \\
\operatorname{div} u=0,\left.\quad u\right|_{S}=b,\left.\quad u\right|_{\infty}=e^{1}=(1,0)
\end{gathered}
$$

во внешней области $D \subset \mathbb{R}^{2}$ с гранищей $\partial D=S$, где $u-$ скорость жидкости, $p-$ давление, $2 \varepsilon$ - число Рейнольдса.

В задаче обтекания $b=0$, но представляют интерес и неоднородные граничные условия.

Отметим, что рассматриваемая задача является особой в том смысле, что, несмотря на наличие малого параметра лишь при младших производных, решение невозможно построить в виде ряда по степеням $\varepsilon$, так как получающаяся в первом приближении система Стокса не имеет решения с нулевым граничным условием и указанным условием на бесконечности (парадокс Стокса).

В случае нулевых граничных условий для внешности круга задача рассматривалась во многих работах (см., например, [1]), где применялся метод сращиваемых асимптотических разложений: растушее решение системы Стокса с нулевым граничным условием срашивалось с решением линеаризованной системы Озеена.

Далее согласование асимптотических разложений реализуется в иной форме. Сначала строится двухпараметрическое решение полной системы Озеена, рассматриваемой в области $\mathbb{R}^{2} \backslash\{0\}$ (т.е. допускаются особенности в нуле типа особенностей фундаментального решения линеаризованной системы Озеена). Затем для выполнения заданных граничных условий к указанному решению добавляется решение системы Стокса с неоднородными граничными условиями. Из условий разрешимости (условий сушествования решения системы Стокса с нулевым значением

Работа выполнена при поддержке РФФИ и гранта Президента РФ для поддержки научных школ НШ-1768.2003.1.

(С) Л.И. САЗОНов, 2003 
на бесконечности) однозначно определяется зависимость двух параметров, входящих в граничные условия, от числа Рейнольдса.

Отметим, что вопрос о сушествовании решения задачи обтекания при любых числах Рейнольдса остается открытым; при малых числах Рейнольдса существование решения установлено в [2]. Ниже этот результат получается в процессе обоснования асимптотики, осушествляемого в соответствии с идеями, изложенными в работе [3].

Настояшая работа состоит из двух параграфов. В 11 изложены необходимые элементы линейной теории, возможно, представляющие и самостоятельный интеpec, $\S 2$ посвящен построению и обоснованию асиптотического разложения.

\section{$\S 1$. Линеаризованная система Озеена в пространстве $\mathbb{R}^{2}$}

Исследуем линеаризованную систему Озеена

$$
\begin{gathered}
\Delta u-2 \varepsilon \frac{\partial}{\partial x_{1}} u-\nabla q=f, \\
\operatorname{div} u=0,
\end{gathered}
$$

которая получается при линеаризации системы Навье-Стокса на постоянном решении $u=(1,0)$.

1.1. Фундаментальное решение и некоторые его оценки. Фундаментальным решением линеаризованной системы Озеена называется набор распределений $\left(u_{\varepsilon}^{k}, q_{\varepsilon}^{k}\right), k=1,2$, являюшийся решением системы

$$
\begin{gathered}
\Delta u_{\varepsilon}^{k}-2 \varepsilon \frac{\partial}{\partial x_{1}} u_{\varepsilon}^{k}-\nabla q_{\varepsilon}^{k}=e^{k} \delta(x), \\
\operatorname{div} u_{\varepsilon}^{k}=0
\end{gathered}
$$

где $\delta(x)-\delta$-распределение Дирака, $e^{k}-$ единичный орт оси $x_{k}$. Распределения $\left(u_{\varepsilon}^{k}, q_{\varepsilon}^{k}\right), k=1,2$, имеют вид (см., например, [4])

$$
\begin{aligned}
q_{\varepsilon}^{k} & =\frac{1}{2 \pi} \frac{\partial}{\partial x_{k}} \ln \frac{1}{|x|}, \\
u_{\varepsilon}^{1} & =\frac{1}{2 \pi}\left[-e^{\varepsilon x_{1}} K_{0}(|\varepsilon x|) e^{1}+\frac{\nabla}{2 \varepsilon}\left(\ln |x|+e^{\varepsilon x_{1}} K_{0}(|\varepsilon x|)\right)\right], \\
u_{\varepsilon, 2}^{2} & =-\frac{1}{4 \pi \varepsilon} \frac{\partial}{\partial x_{1}}\left(\ln |x|+e^{\varepsilon x_{1}} K_{0}(|\varepsilon x|)\right), \\
u_{\varepsilon, 2}^{1} & =u_{\varepsilon, 1}^{2},
\end{aligned}
$$

где

$$
K_{0}(r)=\sum_{k=0}^{\infty} \frac{r^{2 k}}{2^{2 k}(k !)^{2}}\left[\ln \frac{2}{r}+c_{k}\right]
$$

- функция Макдональда.

В терминах преобразования Фурье $\hat{g}(\xi)=\frac{1}{2 \pi} \int_{\mathbb{R}^{2}} g(x) \exp \{-i(x, \xi)\} d x$ имеем

$$
\hat{q}_{\varepsilon}^{k}=\frac{i \xi_{k}}{2 \pi|\xi|^{2}}, \quad \hat{u}_{\varepsilon}^{k}=-\frac{1}{2 \pi}\left(e^{k}-\frac{\xi \xi_{k}}{|\xi|^{2}}\right) \frac{1}{|\xi|^{2}+2 i \varepsilon \xi_{1}} .
$$


Используя (1.3), можно получить разложение фундаментального решения в ряд по $\varepsilon^{k}, \varepsilon^{k} \ln 2 / \varepsilon$ :

$$
u_{\varepsilon, j}^{k}=\ln \frac{2}{\varepsilon} u_{j,-1}^{k}+u_{j, 0}^{k}+\varepsilon \ln \frac{2}{\varepsilon} u_{j, 1}^{k}+\varepsilon u_{j, 2}^{k}+\cdots,
$$

где матрицы $u_{l}=\left(u_{j, l}^{k}\right)_{k, j=1}^{2}$ имеют вид

$$
\begin{aligned}
& u_{-1}=\frac{1}{4 \pi}\left(\begin{array}{cc}
-1 & 0 \\
0 & -1
\end{array}\right), \\
& u_{0}=-\frac{1}{4 \pi}\left(\begin{array}{cc}
\ln \frac{1}{|x|}+\frac{x_{1}^{2}}{|x|^{2}}+c_{0} & \frac{x_{1} x_{2}}{|x|^{2}} \\
\frac{x_{1} x_{2}}{|x|^{2}} & \ln \frac{1}{|x|}+\frac{x_{2}^{2}}{|x|^{2}}+c_{0}-1
\end{array}\right) \text {, } \\
& u_{1}=\frac{1}{8 \pi}\left(\begin{array}{cc}
-x_{1} & x_{2} \\
x_{2} & -3 x_{1}
\end{array}\right) \text {. }
\end{aligned}
$$

Исключая постоянные слагаемые в $u_{0}$, получаем фундаментальное решение системы Стокса

$$
p^{k}=\frac{1}{2 \pi} \frac{\partial}{\partial x_{k}} \ln \frac{1}{|x|}, \quad v_{j}^{k}=-\frac{1}{4 \pi}\left(\delta_{k, j} \ln \frac{1}{|x|}+\frac{x_{k} x_{j}}{|x|^{2}}\right) .
$$

Далее для фундаментального решения при $\varepsilon=1$ будем использовать обозначение $\left(u^{k}, q^{k}\right)$. Существует простая связь: $u_{\varepsilon}^{k}(x)=u^{k}(\varepsilon x), q_{\varepsilon}^{k}(x)=q^{k}(x)$.

Для получения $L_{p}$-оценок фундаментального решения заметим, что

$$
\partial^{\alpha} u_{\varepsilon, j}^{k}(x)=\varepsilon^{|\alpha|}\left(\partial^{\alpha} u_{j}^{k}\right)(\varepsilon x) .
$$

Поэтому

$$
\left\|\partial^{\alpha} u_{\varepsilon, j}^{k}\right\|_{L_{p}\left(\mathbb{R}^{2}\right)}=\varepsilon^{|\alpha|-2 / p}\left\|u_{j}^{k}\right\|_{L_{p}\left(\mathbb{R}^{2}\right)}
$$

и достаточно установить принадлежность пространству $L_{p}\left(\mathbb{R}^{2}\right)$ фундаментального решения при $\varepsilon=1$. В окрестности нуля функция $\left|\partial^{\alpha} u_{j}^{k}(x)\right|$ ограничена функциями $c \ln (1 /|x|)$ при $|\alpha|=0$ и $c|x|^{-|\alpha|}$ при $|\alpha|>0$, в окрестности бесконечности оценки получаются из асимптотики функции Макдональда. В результате устанавливаем, что

$$
\left\|u_{\varepsilon, j}^{k}\right\|_{L_{p}\left(\mathbb{R}^{2}\right)} \leqslant c \varepsilon^{-2 / p},
$$

где $p \in(3, \infty)$, если $(k, j)=(1,1) ; p \in(2, \infty)$, если $(k, j) \neq(1,1)$;

$$
\left\|\frac{\partial}{\partial x_{s}} u_{\varepsilon, j}^{k}\right\|_{L_{p}\left(\mathbb{R}^{2}\right)} \leqslant c \varepsilon^{1-2 / p},
$$

где $p \in(3 / 2,2)$, если $(k, j, s)=(1,1,2) ; p \in(1,2)$, если $(k, j, s) \neq(1,1,2)$.

Далее потребуются оценки производных фундаментального решения вне окрестности нуля. Имеем

$$
\left\|\partial^{\alpha} u_{\varepsilon, j}^{k}\right\|_{L_{p}(|x|>1)}=\varepsilon^{|\alpha|-2 / p}\left\|\partial^{\alpha} u_{j}^{k}\right\|_{L_{p}(|x|>\varepsilon)} .
$$


Учитывая особенность в нуле, получаем

$$
\left\|\partial^{\alpha} u_{\varepsilon, j}^{k}\right\|_{L_{p}(|x|>1)} \leqslant c \theta(\varepsilon,|\alpha|, p)+\varepsilon^{|\alpha|-2 / p}\left\|\partial^{\alpha} u_{j}^{k}\right\|_{L_{p}(|x|>1)},
$$

где $c$ не зависит от $\varepsilon$, функция $\theta$ определяется соотношениями

$$
\theta(\varepsilon,|\alpha|, p)=\left\{\begin{array}{lll}
\left|\frac{\varepsilon^{|\alpha| p-2}-1}{|\alpha| p-2}\right|^{1 / p} & \text { при } & |\alpha| p \neq 2, \\
(\ln 1 / \varepsilon)^{1 / p} & \text { при } & |\alpha| p=2 .
\end{array}\right.
$$

В частности,

$$
\left\|\frac{\partial}{\partial x_{s}} u_{\varepsilon, j}^{k}\right\|_{L_{p}(|x|>1)} \leqslant c \theta(\varepsilon, 1, p)
$$

где $p \in(3 / 2, \infty)$, если $(k, j, s)=(1,1,2) ; p \in(1, \infty)$, если $(k, j, s) \neq(1,1,2)$;

$$
\left\|\partial^{2} u_{\varepsilon, j}^{k}\right\|_{L_{p}(|x|>1)} \leqslant c \theta(\varepsilon, 2, p)
$$

при $1<p<\infty$.

ЗАмЕчАнИЕ 1.1. Функция $\theta$ введена для обеспечения равномерности оценок по $\varepsilon$. Более грубо было бы использовать оценку

$$
\theta(\varepsilon,|\alpha|, p) \leqslant c\left(\ln \frac{1}{\varepsilon}\right)^{1 / p} \begin{cases}\varepsilon^{|\alpha|-2 / p}, & |\alpha| p-2<0 \\ 1, & |\alpha| p-2 \geqslant 0\end{cases}
$$

так как на самом деле логарифмический множитель появляется лишь в окрестности точки $p=2 /|\alpha|$.

Условимся использовать далее обозначение $c$ для различных констант, не зависящих от $\varepsilon$, хотя они могут зависеть от других аргументов. В частности, константы в оценках норм зависят от показателя $p$ и стремятся к бесконечности при приближении к границам интервалов изменения $p$.

В дальнейшем важную роль будут играть операторы свертки

$$
\partial^{\alpha} U_{\varepsilon, j}^{k} f=\partial^{\alpha} u_{\varepsilon, j}^{k} * f .
$$

Из теорем о свертках [5] при $|\alpha|<2$ и мультипликаторах преобразования $Ф$ урье [6], примененных при $|\alpha|=2$ к равенствам (1.5), вытекает справедливость неравенств

$$
\left\|\partial^{\alpha} U_{\varepsilon, j}^{k} f\right\|_{L_{q}\left(\mathbb{R}^{2}\right)} \leqslant c \varepsilon^{|\alpha|-2+2(1 / p-1 / q)}\|f\|_{L_{p}\left(\mathbb{R}^{2}\right)},
$$

если только выполняются условия:

1) $1 \leqslant p \leqslant q \leqslant \infty$ при $|\alpha|<2$ и величина $r$, определяемая соотношением $1 / r=1-1 / p+1 / q$, должна удовлетворять включениям:

a) при $|\alpha|=0$

$$
\begin{aligned}
& r \in(3, \infty), \text { если }(k, j)=(1,1), \\
& r \in(2, \infty), \text { если }(k, j) \neq(1,1) ;
\end{aligned}
$$

б) при $|\alpha|=1$

$$
\begin{array}{lll}
r \in(3 / 2,2), & \text { если } & \left(k, j, \alpha_{1}, \alpha_{2}\right)=(1,1,0,1), \\
r \in(1,2), & \text { если } & \left(k, j, \alpha_{1}, \alpha_{2}\right) \neq(1,1,0,1) ;
\end{array}
$$

2) $1<p=q<\infty$ при $|\alpha|=2$. 
1.2. Гидродинамические потенциалы системы Озеена. Пусть $v, u-$ гладкие соленоидальные поля, $p, q$ - гладкие функции, определенные на плоскости $\mathbb{R}^{2}, T_{i j}(v)=-\delta_{i j} p+v_{i j}-$ тензор напряжений, $v_{i j}=\frac{\partial v_{i}}{\partial x_{j}}+\frac{\partial v_{j}}{\partial x_{i}}, \Omega-$ область с гладкой гранищей $\partial \Omega=S$.

Тогда имеют место формулы Грина

$$
\begin{aligned}
\int_{\Omega}\left(\Delta v_{i}\right. & \left.-2 \varepsilon \frac{\partial v_{i}}{\partial x_{1}}-\frac{\partial p}{\partial x_{i}}\right) u_{i} d x \\
= & -\int_{\Omega}\left(\frac{1}{2} u_{i k} v_{i k}+2 \varepsilon \frac{\partial v_{i}}{\partial x_{1}} u_{i}\right) d x+\int_{S} T_{i k}(v) u_{i} n_{k} d s \\
\int_{\Omega}\left[\left(\Delta v_{i}\right.\right. & \left.\left.-2 \varepsilon \frac{\partial v_{i}}{\partial x_{1}}-\frac{\partial p}{\partial x_{i}}\right) u_{i}-\left(\Delta u_{i}+2 \varepsilon \frac{\partial u_{i}}{\partial x_{1}}+\frac{\partial q}{\partial x_{i}}\right) v_{i}\right] d x \\
= & \int_{S}\left(T_{i k}(v) u_{i} n_{k}-T_{i k}^{\prime}(u) v_{i} n_{k}-2 \varepsilon n_{1} v_{i} u_{i}\right) d s
\end{aligned}
$$

где $n=\left(n_{1}, n_{2}\right)$ - вектор внешней нормали на $S, T_{i k}^{\prime}(u)=\delta_{i k} q+u_{i k}$, причем здесь и далее по повторяюшимся индексам осушествляется суммирование.

Полагая во второй формуле Грина $u=u_{\varepsilon}^{k}(x-y), q=q^{k}(x-y)$, выбирая в качестве $v=v(y), p=p(y)$ гладкое решение неоднородной системы Озеена и интегрируя по $y$, получаем интегральные представления решения $v, p$ :

$$
\begin{aligned}
v_{k}(x)= & \int_{\Omega} u_{\varepsilon, i}^{k}(x-y) f_{i}(y) d y-\int_{S} T_{i j}(v(y)) u_{\varepsilon, i}^{k}(x-y) n_{j}(y) d s_{y} \\
& +\int_{S}\left(T_{i j}^{\prime}\left(u_{\varepsilon}^{k}(x-y)\right)_{y}+2 \varepsilon \delta_{1 j} u_{\varepsilon, i}^{k}(x-y)\right) n_{j}(y) v_{i}(y) d s_{y}, \\
p(x)= & \int_{\Omega} q^{k}(x-y) f_{k}(y) d y-\int_{S} q^{i}(x-y) T_{i j}(v(y)) n_{j}(y) d s_{y} \\
& +\int_{S}\left(-2 \frac{\partial}{\partial x_{j}} q^{i}(x-y)-2 \varepsilon q^{1}(x-y) \delta_{i j}+2 \varepsilon q^{i}(x-y) \delta_{1 j}\right) n_{j}(y) v_{i}(y) d s_{y}
\end{aligned}
$$

(здесь $T_{i j}^{\prime}(\ldots)_{y}$ означает, что дифференширование осушествляется по $y$ ).

На основании (1.17) и (1.18) введем следуюшие гидродинамические потенциалы для системы Озеена:

- объемный потенциал

$$
\begin{aligned}
\left(U_{\varepsilon} f\right)_{k}(x) & =\int_{\mathbb{R}^{2}} u_{\varepsilon, i}^{k}(x-y) f_{i}(y) d y \\
(P f)(x) & =\int_{\mathbb{R}^{2}} q^{k}(x-y) f_{k}(y) d y
\end{aligned}
$$

- потенциал простого слоя

$$
\begin{gathered}
\left(V_{\varepsilon} \psi\right)_{k}(x)=-\int_{S} u_{\varepsilon, i}^{k}(x-y) \psi_{i}(y) d s_{y} \\
(Q \psi)(x)=-\int_{S} q^{i}(x-y) \psi_{i}(y) d s_{y}
\end{gathered}
$$


- потенщиал двойного слоя

$$
\begin{gathered}
\left(W_{\varepsilon} \varphi\right)_{k}(x)=\int_{S}\left(T_{i j}^{\prime}\left(u_{\varepsilon}^{k}(x-y)\right)_{y}+\varepsilon \delta_{1 j} u_{\varepsilon, i}^{k}(x-y)\right) n_{j}(y) \varphi_{i}(y) d s_{y} \\
(\Pi \varphi)(x)=\int_{S}\left(-2 \frac{\partial}{\partial x_{j}} q^{i}(x-y)-2 \varepsilon q^{1}(x-y) \delta_{i j}\right. \\
\left.+\varepsilon q^{i}(x-y) \delta_{1 j}\right) n_{j}(y) \varphi_{i}(y) d s_{y}
\end{gathered}
$$

С помощью введенных потенциалов формулы для $v$ и $p$ представляются в виде

$$
\begin{aligned}
& v=U_{\varepsilon} f+W_{\varepsilon} v+V_{\varepsilon}\left(T(v) n-\varepsilon\left(e^{1}, n\right) v\right), \\
& p=P f+\Pi v+Q\left(T(v) n-\varepsilon\left(e^{1}, n\right) v\right) .
\end{aligned}
$$

ЗАмечАниЕ 1.2. При $\varepsilon=0$ система Озеена переходит в систему Стокса, поэтому формулы Грина для системы Стокса получаются из соответствуюших формул для системы Озеена при $\varepsilon=0$. Однако непосредственный предельный переход возможен только для производных фундаментального решения и, следовательно, только для потенциала двойного слоя. Объемньй потенциал и потенциал простого слоя для системы Стокса получаются из формул $(1.19),(1.20)$ заменой $u_{\varepsilon, j}^{k}$ фундаментальным решением системы Стокса $v_{j}^{k}$.

Далее для потенщиалов простого и двойного слоев для системы Стокса будем использовать обозначения $W$ и $V$.

Предельные соотношения для потенциалов Озеена аналогичны соответствующим сотношениям для потенциалов Стокса [8].

Пусть $\varphi$ - непрерьвное поле на $S$; тогда прямое значение потенщиала двойного слоя $\left(\bar{W}_{\varepsilon} \varphi\right)(x), x \in S$, непрерывно на $S$ и существуют непрерывные предельные значения потенциала $\left(W_{\varepsilon} \varphi\right)(x)$ на $S$ внутри и вне $S$, обозначаемые соответственно $\left(W_{\varepsilon} \varphi\right)_{(i)}(x)$ и $\left(W_{\varepsilon} \varphi\right)_{(e)}(x)$, причем выполняются соотношения

$$
\underset{\left(W_{\varepsilon} \varphi\right)_{(i)}^{(e)}}{(x)}= \pm \frac{1}{2} \varphi(x)+\left(\bar{W}_{\varepsilon} \varphi\right)(x), \quad x \in S .
$$

Аналогично, если $\psi$ - непрерывное поле на $S$, то потенщиал простого слоя $\left(V_{\varepsilon} \psi\right)(x)$ непрерывен на всем пространстве $\mathbb{R}^{2}$ и справедливы предельные соотношения

$$
M_{i j}\left(\varepsilon, V_{\varepsilon} \psi\right)_{(i)}^{(i)}(x) n_{j}(x)= \pm \frac{1}{2} \psi_{i}(x)+\bar{M}_{i j}\left(\varepsilon, V_{\varepsilon} \psi\right)(x) n_{j}(x), \quad x \in S,
$$

где $M_{i j}(\varepsilon, v)=T_{i j}(v)-\varepsilon \delta_{1 j} v_{i}$, причем предельные значения и прямое значение непрерывны на $S$.

1.3. Единственность решений краевых задач для системы Озеена. Пусть $v \in C^{2}(\Omega), p \in C^{1}(\Omega)$ - решение однородной системы Озеена. Рассмотрим два типа краевых условий:

1) условие типа Дирихле $\left.v\right|_{\partial \Omega}=0$,

2 ) условие типа Неймана $\left.M_{i j}(\varepsilon, v) n_{j}\right|_{\partial \Omega}=0$, добавляя в случае внешней области условие на бесконечности: $\left.v\right|_{\infty}=0$. 
Если $\Omega$ - внутренняя область, то, применяя к ее внутренней $\delta$-подобласти $\Omega_{\delta}=$ $\{x \in \Omega, \varrho(x, \partial \Omega)>\delta>0\}$ первую формулу Грина и полагая $u=v$, получаем

$$
\frac{1}{2} \int_{\Omega_{\delta}} v_{i k} v_{i k} d x=\int_{\partial \Omega_{\delta}} M_{i k}(\varepsilon, v) v_{i} n_{k} d s
$$

Предполагая, что

$$
\lim _{\delta \rightarrow 0} \int_{\partial \Omega_{\delta}} M_{i k}(\varepsilon, v) v_{i} n_{k} d s=0,
$$

устанавливаем, что $v_{i k}=0, i, k=1,2$, и, следовательно,

$$
v=\left(c_{1}+c_{3} x_{2}, c_{2}-c_{3} x_{1}\right),
$$

где $c_{i}$ - произвольные постоянные.

Поэтому если $\left.v\right|_{\partial \Omega}=0$, то $v=0$ в $\Omega$, если же выполняется условие 2 ), то из системы Озеена вытекает, что $p=c+2 \varepsilon c_{3} x_{2}$, а само условие 2) принимает вид $\left.\left(p n+\varepsilon n_{1} v\right)\right|_{\partial \Omega}=0$. Отсюда получаем, что при $\varepsilon \neq 0$ система имеет лишь тривиальное решение $v=0$, а при $\varepsilon=0$ ее общее решение имеет вид (1.28) при $p=0$.

В случае внешней области $\Omega$ формула

$$
\frac{1}{2} \int_{\Omega_{\delta}} v_{i k} v_{i k} d x=-\int_{\partial \Omega_{\delta}} M_{i k}(\varepsilon, v) v_{i} n_{k} d s
$$

справедлива при условии

$$
\lim _{R \rightarrow \infty} \int_{|x|=R} M_{i k}(\varepsilon, v) v_{i} n_{k} d s=0 .
$$

Если выполнены соотношения (1.27), (1.30), то для граничных условий 1) или 2) вместе с условием на бесконечности $\left.v\right|_{\infty}=0$ система Озеена имеет лишь тривиальное решение.

1.4. Интегральные уравнения. Рассмотрим следующие задачи:

1) для внешней области $\Omega=\Omega_{e}$ :

$$
\begin{gathered}
\Delta v-2 \varepsilon \frac{\partial v}{\partial x_{1}}-\nabla p=0 \\
\operatorname{div} v=0,\left.\quad v\right|_{\infty}=0,\left.\quad v\right|_{\partial \Omega}=a \in C(\partial \Omega) ;
\end{gathered}
$$

2) для внутренней области $\Omega=\Omega_{i}$ :

$$
\begin{gathered}
\Delta v+2 \varepsilon \frac{\partial v}{\partial x_{1}}-\nabla p=0 \\
\operatorname{div} v=0,\left.\quad M_{i j}(-\varepsilon, v) n_{j}\right|_{\partial \Omega}=b_{i} \in C(\partial \Omega) .
\end{gathered}
$$

Решение задачи 1 ) будем искать в виде потенциала двойного слоя $v=W_{\varepsilon} \varphi$, $\varphi \in C(\partial \Omega)$, а решение задачи 2$)$ - в виде потенциала простого слоя $v=V_{-\varepsilon} \psi$, $\psi \in C(\partial \Omega)$. 
Для определения $\varphi$ и $\psi$ в силу предельных соотношений имеем интегральные уравнения

$$
\begin{aligned}
-\frac{1}{2} \varphi+\bar{W}_{\varepsilon} \varphi & =a, \\
\frac{1}{2} \psi_{k}+\bar{M}_{k j}\left(-\varepsilon, V_{-\varepsilon} \psi\right) n_{j} & =b_{k}, \quad k=1,2,
\end{aligned}
$$

которые, как легко проверяется, взаимно сопряжены.

Покажем, что при $\varepsilon \neq 0$ эти уравнения однозначно разрешимы. Для этого достаточно установить, что хотя бы одно из однородных уравнений имеет лишь тривиальное решение. Пусть $\psi$ - решение уравнения (1.34) при $b=0$. Тогда $v=$ $V_{-\varepsilon} \psi$ является решением задачи 2$)$ при $b=0$. В силу непрерьвности потенциала простого слоя и его предельных значений вьполняется условие единственности и, следовательно, $V_{-\varepsilon} \psi=0$ в $\Omega_{i}$. С другой стороны, $v=V_{-\varepsilon} \psi$ является решением в $\Omega_{e}$ задачи 1$)$ при $a=0$ с заменой $\varepsilon$ на $-\varepsilon$. Используя асимптотику функции $K_{0}$, легко установить выполнение условий единственности из п. 1.3. Поэтому $V_{-\varepsilon} \psi=0$ и в $\Omega_{e}$, но тогда из предельных соотношений вытекает, что $\psi=0$.

Этим завершается доказательство однозначной разрешимости при $\varepsilon \neq 0$.

В случае $\varepsilon=0$, т.е. для системы Стокса [4], однородные уравнения имеют по три линейно независимых решения, которые обозначим через $\varphi^{i}$ для уравнения задачи Дирихле и через $\psi^{i}$ для сопряженного уравнения, причем эти решения можно выбрать так, чтобы выполнялись условия ортогональности

$$
\left(\varphi^{i}, \psi^{j}\right)=\int_{\partial \Omega} \varphi^{i} \psi^{j} d s=\delta_{i j},
$$

при этом $\varphi^{1}=e^{1}, \varphi^{2}=e^{2}, \varphi^{3}=V \psi^{3}$.

Отметим, что приведенные условия однозначно определяют базисы ядер уравнений $\left(\varphi^{3}, \psi^{3}\right.$ с точностью до знака). Напомним также, что $V \psi^{j}=\left(c_{1}^{j}+c_{3}^{j} x_{2}\right.$, $\left.c_{2}^{j}-c_{3}^{j} x_{1}\right)$ во внутренней области $\Omega_{i}$ и в силу выбора базисов $c_{3}^{3} \neq 0$.

Имеет место следуюшее утверждение о парадоксе Стокса.

Внешняя задача

$$
\begin{aligned}
\Delta u-\nabla p & =0, \\
\operatorname{div} u=0,\left.\quad u\right|_{\partial \Omega} & =f \in C(\partial \Omega)
\end{aligned}
$$

имеет единственное решение в классе потенциалов вида

$$
u=c_{1} \varphi^{1}+c^{2} \varphi^{2}+c_{3} V \psi^{3}+W \varphi .
$$

Действительно, для определения плотности $\varphi$ и констант $c_{i}$ имеем интегральное уравнение

$$
-\frac{1}{2} \varphi+W \varphi=f-c_{1} \varphi^{1}-c_{2} \varphi^{2}-c_{3} \varphi^{3} .
$$

Из условий разрешимости однозначно определяются $c_{j}=\left(f, \psi^{j}\right)$, при таком выборе констант уравнение разрешимо и его общее решение имеет вид

$$
\varphi=\alpha_{1} \varphi^{1}+\alpha_{2} \varphi^{2}+\alpha_{3} \varphi^{3}+\varphi_{0},
$$


где $\varphi_{0}$ принадлежит прямому дополнению к ядру $\left\{\varphi^{i}\right\}$ и этим условием определяется однозначно. Тем не менее, решение

$$
v=W \varphi+c_{1} \varphi^{1}+c_{2} \varphi^{2}+c_{3} V \psi^{3}
$$

единственно, так как $W \varphi^{i}=0$ в $\Omega_{e}$.

1.5. $L_{p}$-оценки потенциалов. Ниже приводятся $L_{p}$-оценки потенциалов двойного и простого слоев и их производных.

ТЕОРемА 1.1. Для внешней области $\Omega$ справедливы следующие оценки:

$$
\left\|\left(W_{\varepsilon} \varphi\right)_{k}\right\|_{L_{q_{k}}(\Omega)} \leqslant c \theta\left(\varepsilon, 1, q_{k}\right)\|\varphi\|_{L_{p}(\partial \Omega)}
$$

әде $3<q_{1}<\infty, 2<q_{2}<\infty, q_{k} \leqslant 2 p$;

$$
\left\|W_{\varepsilon} \varphi\right\|_{W_{q}^{2}\left(\Omega^{\prime}\right)} \leqslant c\|\varphi\|_{W_{q}^{2-1 / q}(\partial \Omega)}
$$

где $1<q<\infty, \Omega^{\prime}-$ ограниченная подобласть в $\Omega$;

$$
\left\|\partial^{\alpha} W_{\varepsilon} \varphi\right\|_{L_{q}(|x|>R)} \leqslant c\|\varphi\|_{L_{1}(\partial \Omega)}
$$

где $\partial \Omega \subset\{|x|<R\}, \quad|\alpha| \geqslant 1$, причем $3 / 2<q<\infty$, если $\partial^{\alpha}=\frac{\partial}{\partial x_{2}}, \quad 1<q \leqslant \infty$ в остальных случаях.

ДокАЗАТЕльство. В окрестности границы ядро оператора $W_{\varepsilon}$ равномерно оценивается через $|x-y|^{-1}$. Используя результаты об ограниченности потенциалов в случае разных измерений $[7$, с. 170$]$, получаем, что оператор $W_{\varepsilon}$ равномерно по $\varepsilon$ ограничен из $L_{p}(\partial \Omega)$ в $L_{q}\left(\Omega^{\prime}\right)$ при $1 \leqslant q \leqslant 2 p$.

Вне окрестности границы имеем

$$
\left\|\left(W_{\varepsilon} \varphi\right)_{k}\right\|_{L_{q_{k}}(|x|>R)} \leqslant \sup _{j, y \in \partial \Omega}\left\|W_{\varepsilon, j}^{k}(x, y)\right\|_{L_{q_{k}}(|x|>R)}\|\varphi\|_{L_{1}(\partial \Omega)}
$$

где $W_{\varepsilon, j}^{k}(x, y)$ - ядро потенциала $W_{\varepsilon}$.

Учитывая вид этих ядер, получаем

$$
\begin{aligned}
\left\|W_{\varepsilon, j}^{k}(x, y)\right\|_{L_{q_{k}}(|x|>R) \leqslant} & c\left\{\varepsilon\left\|u_{\varepsilon}^{k}(x-y)\right\|_{L_{q_{k}}(|x|>R)}\right. \\
& \left.+\left\|\nabla u_{\varepsilon}^{k}(x-y)\right\|_{L_{q_{k}}(|x|>R)}+\left\||x-y|^{-1}\right\|_{L_{q_{k}}(|x|>R)}\right\} .
\end{aligned}
$$

Справедливость оценки (1.41) теперь следует из $L_{p}$-оценок фундаментального решения вне окрестности нуля. Аналогично устанавливаются оценки (1.43). Ограниченность оператора $W_{\varepsilon}$ из $W_{q}^{2-1 / q}(\partial \Omega)$ в $W_{q}^{2}\left(\Omega^{\prime}\right)$ доказывается так же, как и в $[8$, с. 78-82] для потенциала двойного слоя системы Стокса. 
Теорема 1.2. Для оператора потенциала простого слоя справедливы оченки

$$
\left\|\left(V_{\varepsilon} \varphi\right)_{k}\right\|_{L_{q_{k}}(\Omega)} \leqslant c \varepsilon^{-2 / q_{k}}\|\varphi\|_{L_{1}(\partial \Omega)},
$$

әде $3<q_{1}<\infty, 2<q_{2}<\infty$;

$$
\left\|\frac{\partial}{\partial x_{s}}\left(V_{\varepsilon} \varphi\right)_{k}\right\|_{L_{q}(\Omega)} \leqslant c \theta(\varepsilon, 1, q)\|\varphi\|_{L_{p}(\partial \Omega)}
$$

для $q \leqslant 2 p$, причем $3 / 2<q<\infty$ при $(k, s)=(1,2), 1<q<\infty$ при $(k, s) \neq(1,2)$;

$$
\left\|\partial^{2}\left(V_{\varepsilon} \varphi\right)_{k}\right\|_{L_{q}(\Omega)} \leqslant c\left(\theta(\varepsilon, 2, q)\|\varphi\|_{L_{1}(\partial \Omega)}+\|\varphi\|_{W_{q}^{1-1 / q}(\partial \Omega)}\right),
$$

где $1<q<\infty$.

Для оббемного потенциала справедливы следующие оценки:

$$
\left\|U_{\varepsilon}\right\|_{L_{p}(\Omega) \rightarrow L_{\infty}(\partial \Omega)} \leqslant c \varepsilon^{-2+2 / p}
$$

при $1<p<3 / 2$

$$
\left\|\frac{\partial}{\partial x_{s}} U_{\varepsilon}\right\|_{L_{p}(\Omega) \rightarrow L_{q}(\partial \Omega)} \leqslant c \theta\left(\varepsilon, 1, p^{\prime}\right),
$$

где $1 / p^{\prime}+1 / p=1, \quad 1<p<3,1 / q \geqslant 2 / p-1$;

$$
\left\|U_{\varepsilon} f\right\|_{W_{q}^{1-1 / q}(S)} \leqslant c\left(\varepsilon^{-2+2 / p}\|f\|_{L_{p}(\Omega)}+\theta\left(\varepsilon, 1, s^{\prime}\right)\|f\|_{L_{s}(\Omega)}\right)
$$

при $1<p<3 / 2, \quad 1<s<3,1 / s \leqslant 1 / q+1 / 2$;

$$
\left\|U_{\varepsilon} f\right\|_{W_{q}^{2-1 / q}(S)} \leqslant c\left(\varepsilon^{-2+2 / p}\|f\|_{L_{p}(\Omega)}+\|f\|_{L_{q}(\Omega)}\right)
$$

nрu $1<p<3 / 2, \quad 1<q<\infty$;

$$
\left\|\partial_{j} U_{\varepsilon} f\right\|_{W_{q}^{1-1 / q}(S)} \leqslant c\left(\theta\left(\varepsilon, 1, p^{\prime}\right)\|f\|_{L_{p}(\Omega)}+\|f\|_{L_{q}(\Omega)}\right),
$$

где $1<p<\infty$ при $j=1,1<p<3$ при $j=2,1 / p \leqslant 1 / q+1 / 2$.

ДокАЗАТЕЛЬСтво. Оценка (1.44) непосредственно следует из $L_{p}$-оценок фундаментального решения. Оценка (1.45), по существу, установлена в предыдушей теореме. Оценка (1.46) вне границы следует из оценок вторых производных фундаментального решения. В окрестности границы, рассматривая $v=V_{\varepsilon} \varphi-\ln \frac{2}{\varepsilon} V_{1} \varphi$ (оператор $V_{1}$ определяется далее, в формуле (1.65)) как решение системы Стокса с правой частью $2 \varepsilon \frac{\partial}{\partial x_{1}} V_{\varepsilon} \varphi$, получаем

$$
\|v\|_{W_{q}^{2}\left(\Omega^{\prime}\right)} \leqslant c\left(\left\|\varepsilon \frac{\partial}{\partial x_{1}} V_{\varepsilon} \varphi\right\|_{L_{q}\left(\Omega^{\prime}\right)}+\left\|V_{\varepsilon} \varphi-\ln \frac{2}{\varepsilon} V_{1} \varphi\right\|_{W_{q}^{2-1 / q}\left(\partial \Omega^{\prime}\right)}\right) .
$$

Оценивая первую норму, как и в случае оператора двойного слоя, и используя для второй нормы разложение в ряд фундаментального решения и сглаживающее свойство потенциала простого слоя для системы Стокса, имеем

$$
\|v\|_{W_{q}^{2}\left(\Omega^{\prime}\right)} \leqslant c\|\varphi\|_{W_{q}^{1-1 / q}(\partial \Omega)} \text { при } 1<q<\infty .
$$

Остается заметить, что $\partial^{2} v=\partial^{2} V_{\varepsilon} \varphi$. 
Для доказательства (1.47) и (1.48) отметим, что рассматриваемые операторы являются сопряженными к операторам $V_{-\varepsilon}$ и $\frac{\partial}{\partial x_{s}} V_{-\varepsilon}$. Для получения оценок (1.49)-(1.51) используем теорему о следах, согласно которой

$$
\left\|U_{\varepsilon} f\right\|_{W_{q}^{j-1 / q}(S)} \leqslant c \sum_{|\alpha|=0, j}\left\|\partial^{|\alpha|} U_{\varepsilon} f\right\|_{L_{q}\left(\Omega^{\prime}\right)}
$$

где $\Omega^{\prime}$ - ограниченная окрестность $\partial \Omega=S$.

Для вторых производных имеем $\left\|\partial^{2} U_{\varepsilon} f\right\|_{L_{q}\left(\Omega^{\prime}\right)} \leqslant c\|f\|_{L_{q}(\Omega)}$ при $1<q<\infty$.

При $|\alpha|=0,1$ удобно рассмотреть сопряженньй оператор

$$
\left(\partial^{\alpha} U_{\varepsilon}\right)^{*}: L_{q^{\prime}}\left(\Omega^{\prime}\right) \rightarrow L_{p^{\prime}}(\Omega)
$$

который совпадает с оператором $(-1)^{|\alpha|} \partial^{\alpha} U_{-\varepsilon}$. Его оценки вытекают из оценок фундаментального решения вне окрестности нуля и оценок для ограниченных областей.

Рассмотрим теперь потенциал $W_{\varepsilon} \varphi^{j}, j=1,2$. Применяя теорему Остроградского-Гаусса, получаем представление

$$
\left(W_{\varepsilon} \varphi^{j}\right)_{k}(x)=-\varepsilon \int_{\Omega_{i}} \frac{\partial}{\partial y_{1}}\left(u_{\varepsilon, j}^{k}(x-y)\right) d y .
$$

Обратный переход к криволинейному интегралу приводит к представлению $W_{\varepsilon} \varphi^{j}$ в форме потенциала простого слоя:

$$
\left(W_{\varepsilon} \varphi^{j}\right)_{k}(x)=-\varepsilon \int_{\partial \Omega} u_{\varepsilon, j}^{k}(x-y) n_{1}(y) d s_{y} .
$$

Отсюда вытекает следующая оценка:

$$
\left\|W_{\varepsilon} \varphi^{j}\right\|_{L_{q}(\Omega)} \leqslant \varepsilon \theta(\varepsilon, 1, q), \quad j=1,2, \quad 1<q<\infty .
$$

Действительно, для оценки вне границы следует использовать представление (1.53), а в окрестности границы - представление (1.54).

Отметим также, что справедлива оценка

$$
\left\|\partial^{\alpha} W_{\varepsilon} \varphi^{j}\right\|_{L_{q}(\Omega)} \leqslant \varepsilon \theta(\varepsilon,|\alpha|+1, q), \quad|\alpha|=1,2, \quad 1<q<\infty .
$$

Вне окрестности границы следует использовать формулу (1.53), а в ее окрестности - формулу (1.54).

1.6. Разрешающий оператор системы Озеена и его оценки при малых $\varepsilon$. Ниже исследуется зависимость от $\varepsilon$ решения внешней задачи Дирихле для однородного уравнения Озеена при малых $\varepsilon$. Для этого к интегральному уравнению (1.33) применим метод Ляпунова-Шмидта.

Заметим, что при $\varepsilon=0$ уравнение (1.33) является уравнением внешней задачи Дирихле для системы Стокса, при этом оператор $-\frac{1}{2} I+\bar{W}$ и его сопряженньй $\left(-\frac{1}{2} I+\bar{W}\right)^{*}$ имеют трехмерные ядра $N$ и $N^{*}$, их базисы выберем специальным образом, как это указано в п. 1.4, причем ниже используем обозначение $\varphi^{3}=V \psi^{3}=A+\gamma\left(x_{2},-x_{1}\right)$. 
Предварительно рассмотрим случай, когда поле $a$ удовлетворяет условиям разрешимости $\left(a, \psi^{j}\right)=0, j=1,2,3$. Введем проектор

$$
P \psi=\psi-\sum_{i=1}^{3}\left(\psi, \psi^{i}\right) \varphi^{i}
$$

на $\operatorname{Im}\left(-\frac{1}{2} I+\bar{W}\right)$ и его дополнительный проектор $Q=I-P$, который одновременно является проектором на $N$. Применяя проекторы $P$ и $Q$ к уравнению (1.33) и представляя $\varphi$ в виде $\varphi=c_{1} \varphi^{1}+c_{2} \varphi^{2}+c_{3} \varphi^{3}+\xi$, где $Q \xi=0$, сведем его к системе уравнений

$$
\begin{aligned}
& \left(-\frac{1}{2} I+\bar{W}\right) \xi+P\left(\bar{W}_{\varepsilon}-\bar{W}\right)\left(\xi+\sum_{i=0}^{3} c_{i} \varphi^{i}\right)=a, \\
& Q\left(\bar{W}_{\varepsilon}-\bar{W}\right)\left(\xi+\sum_{i=1}^{3} c_{i} \varphi^{i}\right)=0 .
\end{aligned}
$$

Покажем, что второе уравнение однозначно разрешимо относительно $c_{j}$ при любом $\xi$, а затем, подставляя $c_{j}=c_{j}(\xi)$ в первое уравнение, установим его однозначную разрешимость при достаточно малых $\varepsilon \neq 0$.

Предварительно преобразуем к удобной форме поля $\left(\bar{W}_{\varepsilon}-\bar{W}\right) \varphi^{j}$. Для этого заметим, что пары $\varphi^{j}, p=0(j=1,2)$ и $\varphi^{3}, p=2 \varepsilon \gamma x_{2}$ являются решением системы Озеена, и поэтому из интегрального представления решения в $\Omega_{i}$ (см. (1.22)) получаем

$$
\begin{aligned}
& \varphi^{j}=W_{\varepsilon} \varphi^{j}-\varepsilon V_{\varepsilon}\left(n_{1} \varphi^{j}\right), \quad j=1,2, \\
& \varphi^{3}=W_{\varepsilon} \varphi^{3}-\varepsilon V_{\varepsilon}\left(n_{1} \varphi^{3}+2 \gamma n x_{2}\right) .
\end{aligned}
$$

Внутренние предельные соотношения приводят к формулам

$$
\begin{aligned}
& \left(-\frac{1}{2} I+\bar{W}_{\varepsilon}\right) \varphi^{j}-\varepsilon V_{\varepsilon}\left(n_{1} \varphi^{j}\right)=0, \quad j=1,2, \\
& \left(-\frac{1}{2} I+\bar{W}_{\varepsilon}\right) \varphi^{3}-\varepsilon V_{\varepsilon}\left(n_{1} \varphi^{3}+2 \gamma n x_{2}\right)=0 .
\end{aligned}
$$

Из разложения фундаментального решения в ряд имеем следуюшие разложения для потенциалов:

$$
\begin{aligned}
\bar{W}_{\varepsilon} & =\bar{W}+\varepsilon \ln \frac{2}{\varepsilon} W_{1}+\varepsilon W_{2}+\varepsilon^{2} \ln \frac{2}{\varepsilon} W_{3}+\cdots, \\
V_{\varepsilon} & =\ln \frac{2}{\varepsilon} V_{1}+V_{2}+\varepsilon \ln \frac{2}{\varepsilon} V_{3}+\cdots,
\end{aligned}
$$

где операторы $V_{j}, j=1,2,3$, определяются формулами

$$
\begin{gathered}
V_{1} \psi=\frac{1}{4 \pi} \int_{S} \psi d s \\
V_{2} \psi=V \psi+\Lambda V_{1} \psi, \quad \Lambda=\operatorname{diag}\left(c_{0}, c_{0}-1\right) \\
V_{3} \psi(x)=\frac{1}{8 \pi} \int_{S}\left(\begin{array}{cc}
x_{1}-y_{1} & -x_{2}+y_{2} \\
-x_{2}+y_{2} & 3\left(x_{1}-y_{1}\right)
\end{array}\right)\left(\begin{array}{l}
\psi_{1}(y) \\
\psi_{2}(y)
\end{array}\right) d s_{y} .
\end{gathered}
$$


Используя ряды (1.63), (1.64), из соотношений (1.61) и (1.62) получаем

$$
\begin{aligned}
& W_{j}\left(\varphi^{k}\right)=V_{j}\left(n_{1} \varphi^{k}\right), \quad k=1,2, \\
& W_{j}\left(\varphi^{3}\right)=V_{j}\left(n_{1} \varphi^{3}+2 \gamma n x_{2}\right), \quad j=1,2, \ldots
\end{aligned}
$$

Далее, легко проверяется, что

$$
\begin{aligned}
& V_{1}\left(n_{1} \varphi^{k}\right)=0, \quad V_{2}\left(n_{1} \varphi^{k}\right)=V\left(n_{1} \varphi^{k}\right), \quad k=1,2, \\
& V_{3}\left(n_{1} \varphi^{k}\right)=\alpha_{k} \varphi^{k}, \quad k=1,2, \quad \alpha_{1}=-\frac{\mu\left(\Omega_{i}\right)}{8 \pi}, \quad \alpha_{2}=-\frac{3 \mu\left(\Omega_{i}\right)}{8 \pi}, \\
& V_{1}\left(n_{1} \varphi^{3}+2 \gamma n x_{2}\right)=V_{1}\left(\gamma\left(x_{2},-x_{1}\right) n_{1}+2 \gamma n x_{2}\right)=-2 \gamma \alpha_{1} \varphi^{2} \\
& V_{2}\left(n_{1} \varphi^{3}+2 \gamma n x_{2}\right)=V\left(n_{1} \varphi^{3}+2 \gamma n x_{2}\right)-2 \gamma \alpha_{1}\left(c_{0}-1\right) \varphi^{2}
\end{aligned}
$$

Теперь, используя разложение для $\bar{W}_{\varepsilon}$, связь потенциалов двойного и простого слоев для базисных полей (формулы (1.68), (1.69)) и соотношения (1.70)-(1.73), устанавливаем

$$
\begin{aligned}
\left(\bar{W}_{\varepsilon}-\bar{W}\right) & \left(\sum_{i=1}^{3} c_{i} \varphi^{i}\right)=-\varepsilon \ln \frac{2}{\varepsilon} 2 \gamma \alpha_{1} c_{3} \varphi^{2} \\
& +\varepsilon\left(V\left(\sum_{i=1}^{3} c_{i} \varphi^{i} n_{1}+c_{3} 2 \gamma n x_{2}\right)-2 \gamma \alpha_{1}\left(c_{0}-1\right) c_{3} \varphi^{2}\right) \\
& +\varepsilon^{2} \ln \frac{2}{\varepsilon}\left(c_{1} \alpha_{1} \varphi^{1}+c_{2} \alpha_{2} \varphi^{2}+c_{3} V_{3}\left(n_{1} \varphi^{3}+2 \gamma n x_{2}\right)\right)+O\left(\varepsilon^{2}\right) .
\end{aligned}
$$

Отметим также, что справедливы соотношения

$$
\begin{aligned}
& \left(\psi^{3}, V\left(n_{1} \varphi^{i}\right)\right)=-\delta_{i 2} \gamma \mu\left(\Omega_{i}\right), \quad i=1,2 \\
& \left(\psi^{j}, V\left(n_{1} \varphi^{i}\right)\right)=0, \quad i, j=1,2
\end{aligned}
$$

Действительно, имеем

$$
\left(\psi^{3}, V\left(n_{1} \varphi^{i}\right)\right)=\left(V \psi^{3}, n_{1} \varphi^{i}\right)=\left(\gamma\left(x_{2},-x_{1}\right), n_{1} \varphi^{i}\right)=-\delta_{i 2} \gamma \mu\left(\Omega_{i}\right) .
$$

Для доказательства (1.76) рассмотрим поле

$$
w^{i}=V\left(n_{1} \varphi^{i}\right)-b_{1} \varphi^{1}-b_{2} \varphi^{2}-b_{3} V \psi^{3}-W \xi,
$$

где $b_{j}=\left(\psi^{j}, V\left(n_{1} \varphi^{i}\right)\right), j=1,2,3 ; \xi$-решение уравнения

$$
\left(-\frac{1}{2} I+\bar{W}\right) \xi=V\left(n_{1} \varphi^{i}\right)-b_{1} \varphi^{1}-b_{2} \varphi^{2}-b_{3} V \psi^{3} .
$$

Пара $w^{i}, p=Q\left(n_{1} \varphi^{i}-b_{3} \psi^{3}\right)-\Pi \xi$ (здесь $Q$ и П - соответственно операторы простого и двойного слоя для давления) является решением системы Стокса во внешней области и удовлетворяет условиям

$$
\left.w^{i}\right|_{S}=0, \quad|p(x)| \leqslant c|x|^{-2}, \quad\left|T_{k j}\left(w^{i}\right)\right| \leqslant c|x|^{-2}, \quad\left|w^{i}(x)\right| \leqslant c .
$$


Поэтому из результатов, полученных в п. 1.3 , следует, что $w^{i}=0$ в $\Omega_{e}$, и так как $V\left(n_{1} \varphi^{i}\right), V \psi^{3}, W \xi$ стремятся к нулю при $|x| \rightarrow \infty$, то $b_{1}=b_{2}=0$, что и доказывает соотношение (1.76).

С учетом формул (1.74)-(1.76) второе уравнение системы (1.58) приводится к виду

$$
\sum_{j=1}^{3} a_{i j} c_{j}=\left(\eta, \psi^{i}\right), \quad i=1,2,3,
$$

где $\eta=-\left(\bar{W}_{\varepsilon}-\bar{W}\right) \xi$, а коэффициенты $a_{i j}$ разлагаются в ряды по $\varepsilon^{k} \ln \frac{2}{\varepsilon}, \varepsilon^{k}$ и их главные члены имеют вид

$$
\begin{aligned}
& a_{11}=\varepsilon^{2} \ln \frac{2}{\varepsilon} a_{11}^{0}, \quad a_{12}=\varepsilon^{2} a_{12}^{0}, \quad a_{13}=\varepsilon a_{13}^{0}, \\
& a_{21}=\varepsilon^{2} a_{21}^{0}, \quad a_{22}=\varepsilon^{2} \ln \frac{2}{\varepsilon} a_{22}^{0}, \quad a_{23}=\varepsilon \ln \frac{2}{\varepsilon} a_{23}^{0}, \\
& a_{31}=\varepsilon^{2} a_{31}^{0}, \quad a_{32}=\varepsilon a_{32}^{0}, \quad a_{33}=\varepsilon a_{33}^{0},
\end{aligned}
$$

причем $a_{i j}^{0}$ не зависят от $\varepsilon$ и $a_{11}^{0}=\alpha_{1}, a_{22}^{0}=\alpha_{2}, a_{23}^{0}=-2 \gamma \alpha_{1}, a_{32}^{0}=-\gamma \mu\left(\Omega_{i}\right)$ заведомо отличны от нуля.

Для определителей Крамера системы (1.81) имеем следующие выражения:

$$
\begin{gathered}
\Delta=\Delta_{0} \varepsilon^{4}\left(\ln \frac{2}{\varepsilon}\right)^{2}, \\
\Delta_{1}=\Delta_{11}\left(\eta, \psi^{1}\right) \varepsilon^{2} \ln \frac{2}{\varepsilon}+\Delta_{12}\left(\eta, \psi^{2}\right) \varepsilon^{2}+\Delta_{13}\left(\eta, \psi^{3}\right) \varepsilon^{3} \ln \frac{2}{\varepsilon} \\
\Delta_{2}=\Delta_{21}\left(\eta, \psi^{1}\right) \varepsilon^{3} \ln \frac{2}{\varepsilon}+\Delta_{22}\left(\eta, \psi^{2}\right) \varepsilon^{3} \ln \frac{2}{\varepsilon}+\Delta_{23}\left(\eta, \psi^{3}\right) \varepsilon^{3}\left(\ln \frac{2}{\varepsilon}\right)^{2}, \\
\Delta_{3}=\Delta_{31}\left(\eta, \psi^{1}\right) \varepsilon^{3}+\Delta_{32}\left(\eta, \psi^{2}\right) \varepsilon^{3} \ln \frac{2}{\varepsilon}+\Delta_{33}\left(\eta, \psi^{3}\right) \varepsilon^{4}\left(\ln \frac{2}{\varepsilon}\right)^{2},
\end{gathered}
$$

где главные члены $\Delta_{0}, \Delta_{i j}$ суть

$$
\begin{array}{lll} 
& \Delta_{0} \sim-a_{11}^{0} a_{32}^{0} a_{23}^{0} \neq 0, \\
\Delta_{11} \sim-a_{23}^{0} a_{32}^{0}, & \Delta_{12} \sim a_{32}^{0} a_{13}^{0}, & \Delta_{13} \sim a_{12}^{0} a_{23}^{0}-a_{22}^{0} a_{13}^{0}, \\
\Delta_{21} \sim a_{31}^{0} a_{23}^{0}, & \Delta_{22} \sim a_{11}^{0} a_{33}^{0}, & \Delta_{23} \sim-a_{11}^{0} a_{23}^{0}, \\
\Delta_{31} \sim a_{21}^{0} a_{32}^{0}, & \Delta_{32} \sim-a_{11}^{0} a_{32}^{0}, & \Delta_{33} \sim a_{11}^{0} a_{22}^{0} .
\end{array}
$$

Тогда функционалы $c_{j}$ определяются формулами

$$
\begin{aligned}
& c_{1}=\frac{\Delta_{11}\left(\eta, \psi^{1}\right)}{\Delta_{0} \varepsilon^{2} \ln \frac{2}{\varepsilon}}+\frac{\Delta_{12}\left(\eta, \psi^{2}\right)}{\Delta_{0} \varepsilon^{2}\left(\ln \frac{2}{\varepsilon}\right)^{2}}+\frac{\Delta_{13}\left(\eta, \psi^{3}\right)}{\Delta_{0} \varepsilon \ln \frac{2}{\varepsilon}}, \\
& c_{2}=\frac{\Delta_{21}\left(\eta, \psi^{1}\right)}{\Delta_{0} \varepsilon \ln \frac{2}{\varepsilon}}+\frac{\Delta_{22}\left(\eta, \psi^{2}\right)}{\Delta_{0} \varepsilon \ln \frac{2}{\varepsilon}}+\frac{\Delta_{23}\left(\eta, \psi^{3}\right)}{\Delta_{0} \varepsilon}, \\
& c_{3}=\frac{\Delta_{31}\left(\eta, \psi^{1}\right)}{\Delta_{0} \varepsilon\left(\ln \frac{2}{\varepsilon}\right)^{2}}+\frac{\Delta_{32}\left(\eta, \psi^{2}\right)}{\Delta_{0} \varepsilon \ln \frac{2}{\varepsilon}}+\frac{\Delta_{33}\left(\eta, \psi^{3}\right)}{\Delta_{0}} .
\end{aligned}
$$


Применяя к (1.74) проектор $P$, приведем первое уравнение системы (1.58) к виду

$$
\begin{gathered}
\left(-\frac{1}{2}+\bar{W}\right) \xi+P\left(\bar{W}_{\varepsilon}-\bar{W}\right) \xi+\varepsilon P V\left(n_{1} \sum_{i=1}^{3} c_{i} \varphi^{i}+2 \gamma c_{3} n x_{2}\right) \\
+\varepsilon^{2} \ln \frac{2}{\varepsilon} c_{3} P V_{3}\left(n_{1} \varphi^{3}+2 \gamma n x_{2}\right)+\cdots=a
\end{gathered}
$$

Учитывая, что

$$
\eta=-\left(\bar{W}_{\varepsilon}-\bar{W}\right) \xi=-\varepsilon \ln \frac{2}{\varepsilon} W_{1} \xi-\varepsilon W_{2} \xi-\cdots, \quad P V\left(\varphi^{1} n_{1}\right)=V\left(\varphi^{1} n_{1}\right)
$$

(см. (1.75), (1.76)), и подставляя выражения для $c_{j}$ в (1.85), представим уравнение (1.85) в форме

$$
\left(-\frac{1}{2}+\bar{W}\right) \xi-\frac{\Delta_{11}}{\Delta_{0}}\left(W_{1} \xi, \psi^{1}\right) V\left(n_{1} \varphi^{1}\right)+\cdots=a
$$

где остальные члены имеют порядок $O\left(\left(\ln \varepsilon^{-1}\right)^{-1}\right)$.

Покажем, что уравнение (1.86) однозначно разрешимо при достаточно малом $\varepsilon \neq 0$. Поскольку

$$
\frac{\Delta_{11}}{\Delta_{0}}=\frac{1}{a_{11}^{0}}+O\left(\left(\ln \frac{2}{\varepsilon}\right)^{-1}\right)=\frac{1}{\alpha_{1}}+O\left(\left(\ln \frac{2}{\varepsilon}\right)^{-1}\right),
$$

то достаточно установить, что однородное уравнение

$$
\left(-\frac{1}{2}+\bar{W}\right) \xi-\frac{1}{\alpha_{1}}\left(W_{1} \xi, \psi^{1}\right) V\left(n_{1} \varphi^{1}\right)=0
$$

имеет лишь тривиальное решение. Так как для поля $V\left(n_{1} \varphi^{1}\right)$ выполнены условия разрешимости (см. (1.75), (1.76)), то сушествует единственное поле $\psi$, для которого $\left(\psi^{j}, \psi\right)=0, j=1,2,3,\left(-\frac{1}{2}+\bar{W}\right) \psi=V\left(n_{1} \varphi^{1}\right)$. Но тогда решение уравнения (1.87) определяется из уравнения

$$
\xi-\frac{1}{\alpha_{1}}\left(W_{1} \xi, \psi^{1}\right) \psi=0,
$$

и, следовательно, достаточно установить, что

$$
1-\frac{1}{\alpha_{1}}\left(W_{1} \psi, \psi^{1}\right) \neq 0 .
$$

Из формул разложения фундаментального решения следует, что ядро оператора $W_{1}$ не зависит от $x$, поэтому $W_{1} \psi$ - постоянный вектор и, следовательно, $\left(W_{1} \psi, \psi^{1}\right)=\left(W_{1} \psi\right)_{1}$. Проводя вычисления с применением формул $(1.6)-(1.8)$, получим

$$
\left(W_{1} \psi, \psi^{1}\right)=-\frac{1}{4 \pi} \int_{S} n_{2} \psi_{2} d s .
$$

Для нахождения последнего интеграла воспользуемся равенством

$$
V\left(n_{1} \varphi^{1}\right)=W(\psi)
$$


справедливым в $\Omega_{e}$, и вычислим асимптотику этих интегралов при $|x| \rightarrow \infty$. Опуская простые выкладки, имеем

$$
V\left(n_{1} \varphi^{1}\right)_{k}(x) \sim \frac{x_{k}\left(x_{1}^{2}-x_{2}^{2}\right) \mu\left(\Omega_{i}\right)}{4 \pi|x|^{4}} .
$$

С другой стороны,

$$
\begin{aligned}
(W \psi)_{k}(x) & =-\frac{1}{\pi} \int_{S} \frac{\left(x_{k}-y_{k}\right)\left(x_{i}-y_{i}\right)\left(x_{j}-y_{j}\right)}{|x-y|^{4}} \psi_{j}(y) n_{i}(y) d s_{y} \\
& \sim-\frac{1}{\pi} \int_{S} \frac{x_{k} x_{i} x_{j}}{|x|^{4}} \psi_{j}(y) n_{i}(y) d s_{y}=-\frac{x_{k} x_{i} x_{j} \gamma_{i j}}{\pi|x|^{4}}
\end{aligned}
$$

где $\gamma_{i j}=\int_{S} n_{i} \psi_{j} d s$.

Сравнивая полученные асимптотики, устанавливаем, что

$$
\gamma_{11}=-\frac{\mu\left(\Omega_{i}\right)}{4}, \quad \gamma_{22}=-\gamma_{11}, \quad \gamma_{12}=\gamma_{21}=0 .
$$

Следовательно,

$$
\left(W_{1} \psi, \psi^{1}\right)=-\frac{\gamma_{22}}{4 \pi}=-\frac{\mu\left(\Omega_{i}\right)}{16 \pi} .
$$

Поскольку $\alpha_{1}=-\frac{\mu\left(\Omega_{i}\right)}{8 \pi}$, то

$$
1-\frac{1}{\alpha_{1}}\left(W_{1} \psi, \psi^{1}\right)=\frac{1}{2} \neq 0
$$

чем и завершается доказательство однозначной разрешимости.

Решая уравнение (1.86), для $\xi$ получаем оценку

$$
\|\xi\|_{C(S)} \leqslant c\|a\|_{C(S)} .
$$

Отметим, что ввиду сглаживающего действия оператора прямого значения потенциала двойного слоя уравнение однозначно разрешимо во всех пространствах $L_{p}(S)$ и, более того, во всех пространствах Соболева-Слободецкого $W_{p}^{\alpha}(S)$ и выполняются оценки

$$
\|\xi\|_{W_{p}^{\alpha}(S)} \leqslant c\|a\|_{W_{p}^{\alpha}(S)} .
$$

Для функционалов $c_{j}$ ввиду формул (1.82)-(1.84) и (1.91) справедливы оценки

$$
\begin{gathered}
\left|c_{1}\right| \leqslant(c / \varepsilon)\|a\|_{L_{1}(S)}, \quad\left|c_{2}\right| \leqslant c \ln (2 / \varepsilon)\|a\|_{L_{1}(S)}, \\
\left|c_{3}\right| \leqslant c\|a\|_{L_{1}(S)} .
\end{gathered}
$$

Пусть $a$ не удовлетворяет условиям разрешимости $\left(\psi^{j}, a\right)=0, j=1,2,3$. Тогда решение внешней задачи будем искать в форме

$$
v=W_{\varepsilon} \varphi+b_{1} u_{\varepsilon}^{1}+b_{2} u_{\varepsilon}^{2}+b_{3} V_{\varepsilon} \psi^{3},
$$

плотность $\varphi$ определяется из уравнения

$$
\left(-\frac{1}{2} I+\bar{W}_{\varepsilon}\right) \varphi=a-b_{1} u_{\varepsilon}^{1}-b_{2} u_{\varepsilon}^{2}-b_{3} V_{\varepsilon} \psi^{3},
$$


а константы $b_{j}$ выбираются из условий

$$
\left(\psi^{j}, a-b_{1} u_{\varepsilon}^{1}-b_{2} u_{\varepsilon}^{2}-b_{3} V_{\varepsilon} \psi^{3}\right)=0, \quad j=1,2,3 .
$$

Поскольку

$$
\begin{gathered}
u_{\varepsilon}^{j} \sim-\frac{1}{4 \pi} \varphi^{j} \ln \frac{2}{\varepsilon}+O(1), \\
V_{\varepsilon} \psi^{3} \sim V \psi^{3}+O\left(\varepsilon \ln \frac{2}{\varepsilon}\right)=\varphi^{3}+O\left(\varepsilon \ln \frac{2}{\varepsilon}\right),
\end{gathered}
$$

то для определителя системы (1.96) справедливо асимптотическое соотношение

$$
\Delta \sim \frac{1}{16 \pi^{2}}\left(\ln \frac{2}{\varepsilon}\right)^{2}
$$

и главные члены функционалов $b_{j}$ имеют вид

$$
\begin{gathered}
b_{1} \sim-4 \pi\left(a, \psi^{1}\right)\left(\ln \frac{2}{\varepsilon}\right)^{-1}, \\
b_{2} \sim-4 \pi\left(a, \psi^{2}\right)\left(\ln \frac{2}{\varepsilon}\right)^{-1}, \quad b_{3} \sim\left(a, \psi^{3}\right) .
\end{gathered}
$$

Теперь остается применить к уравнению (1.95) предыдущие результаты для случая выполнения условий разрешимости. Таким образом, приходим к следуюшему утверждению.

ТЕОРЕМа 1.3. При достаточно мальх $\varepsilon \neq 0$ решение внешней задачи (1.31) можно представить в виде

$$
v=W_{\varepsilon}\left(c_{1} \varphi^{1}+c_{2} \varphi^{2}+c_{3} \varphi^{3}+\xi\right)+b_{1} u_{\varepsilon}^{1}+b_{2} u_{\varepsilon}^{2}+b_{3} V_{\varepsilon} \psi^{3},
$$

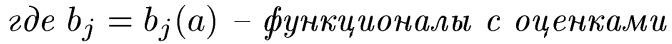

$$
\left|b_{j}\right| \leqslant c\|a\|_{L_{1}(S)}(\ln (2 / \varepsilon))^{-1}, \quad j=1,2, \quad\left|b_{3}\right| \leqslant c\|a\|_{L_{1}(S)},
$$

$c_{j}=c_{j}(a)-$ функциональ с оценками

$$
\left|c_{1}\right| \leqslant c(\varepsilon)^{-1}\|a\|_{L_{1}(S)}, \quad\left|c_{2}\right| \leqslant c \ln (2 / \varepsilon)\|a\|_{L_{1}(S)}, \quad\left|c_{3}\right| \leqslant c\|a\|_{L_{1}(S)},
$$

причем

$$
\|\xi\|_{C(S)} \leqslant c\|a\|_{C(S)}, \quad\|\xi\|_{W_{p}^{\alpha}(S)} \leqslant c\|a\|_{W_{p}^{\alpha}(S)}
$$

Получим $L_{p}$-оценки для $\partial^{\alpha} v$. Из соотношения (1.100) имеем

$$
\begin{aligned}
\left\|\partial^{\alpha} v\right\|_{L_{q}(\Omega)} \leqslant & \sum_{j=1}^{3}\left|c_{j}\right|\left\|\partial^{\alpha} W_{\varepsilon} \varphi^{j}\right\|_{L_{q}(\Omega)}+\left\|\partial^{\alpha} W_{\varepsilon} \xi\right\|_{L_{q}(\Omega)} \\
& +\sum_{j=1}^{2}\left|b_{j}\right|\left\|\partial^{\alpha} u_{\varepsilon}^{j}\right\|_{L_{q}(\Omega)}+\left|b_{3}\right|\left\|\partial^{\alpha} V_{\varepsilon} \psi^{3}\right\|_{L_{q}(\Omega)} .
\end{aligned}
$$


Теперь, используя оценки фундаментального решения и потенциалов, получаем

$$
\left\|v_{k}\right\|_{L_{q_{k}}(\Omega)} \leqslant c\left\{\theta\left(\varepsilon, 1, q_{k}\right)\left(\|a\|_{L_{1}(S)}+\|\xi\|_{L_{q_{k} / 2}(S)}\right)+\varepsilon^{-2 / q_{k}}\|a\|_{L_{1}(S)}\right\},
$$

откуда с учетом оценок $\xi$ имеем

$$
\left\|v_{k}\right\|_{L_{q_{k}}(\Omega)} \leqslant c\left\{\theta\left(\varepsilon, 1, q_{k}\right)\|a\|_{L_{q_{k} / 2}(S)}+\varepsilon^{-2 / q_{k}}\|a\|_{L_{1}(S)}\right\},
$$

где $3<q_{1}<\infty, 2<q_{2}<\infty$;

$$
\left\|\frac{\partial}{\partial x_{s}} v_{k}\right\|_{L_{q}(\Omega)} \leqslant c\left\{(\theta(\varepsilon, 1, q)+\theta(\varepsilon, 2, q))\|a\|_{L_{1}(S)}+\|a\|_{W_{q}^{1-1 / q}(S)}\right\},
$$

где $3 / 2<q<\infty$, если $(k, s)=(1,2)$, и $1<q<\infty$, если $(k, s) \neq(1,2)$;

$$
\left\|\partial^{2} v\right\|_{L_{q}(\Omega)} \leqslant c\left\{\theta(\varepsilon, 2, q)\|a\|_{L_{1}(S)}+\|a\|_{W_{q}^{2-1 / q}(S)}\right\} \text { при } 1<q<\infty .
$$

Рассмотрим неоднородную систему Озеена с однородными граничными условиями

$$
\begin{gathered}
\Delta u-2 \varepsilon \frac{\partial}{\partial x_{1}} u-\nabla p=f, \\
\operatorname{div} u=0,\left.\quad u\right|_{\partial \Omega}=0,\left.\quad u\right|_{\infty}=0 .
\end{gathered}
$$

Оператор $u=R_{\varepsilon} f$, представляющий ее решение, будем называть разрешающим оператором. Его можно представить в виде $R_{\varepsilon}=U_{\varepsilon}+L_{\varepsilon}$, где $U_{\varepsilon}$ - оператор объемного потенщиала, а $v=L_{\varepsilon} f$ - оператор, представляющий решение однородной системы Озеена с неоднородным граничным условием $a=-\left.U_{\varepsilon} f\right|_{\partial \Omega}$. Оценки для $v=L_{\varepsilon} f$ могут быть получены из предыдущих оценок с использованием оценок $\left\|U_{\varepsilon} f\right\|_{W_{q}^{l-1 / q}(S)}{ }^{\text {. }}$

Таким образом, имеем

$$
\left\|\left(L_{\varepsilon} f\right)_{k}\right\|_{L_{q_{k}}(\Omega)} \leqslant c \varepsilon^{-2+2\left(1 / p-1 / q_{k}\right)}\|f\|_{L_{p}(\Omega)},
$$

где $3<q_{1}<\infty, 2<q_{2}<\infty, 1<p<3 / 2$;

$$
\begin{aligned}
\left\|\frac{\partial}{\partial x_{j}}\left(L_{\varepsilon} f\right)_{k}\right\|_{L_{q}(\Omega)} \leqslant & c\left\{(\theta(\varepsilon, 1, q)+\theta(\varepsilon, 2, q)) \varepsilon^{-2+2 / p}\|f\|_{L_{p}(\Omega)}\right. \\
& \left.+\theta\left(\varepsilon, 1, s^{\prime}\right)\|f\|_{L_{s}(\Omega)}\right\},
\end{aligned}
$$

где $3 / 2<q<\infty$, если $(k, j)=(1,2)$, и $1<q<\infty$, если $(k, j) \neq(1,2) ; 1<p<3 / 2$, $1<s<3,1 / s \leqslant 1 / q+1 / 2$;

$$
\left\|\partial^{2} L_{\varepsilon} f\right\|_{L_{q}(\Omega)} \leqslant c\left\{\theta(\varepsilon, 2, q) \varepsilon^{-2+2 / p}\|f\|_{L_{p}(\Omega)}+\|f\|_{L_{q}(\Omega)}\right\}
$$

при $1<q<\infty, 1<p<3 / 2$.

В случае $f=\partial_{j} g$ имеем

$$
\left\|\left(L_{\varepsilon} \partial_{j} g\right)_{k}\right\|_{L_{q_{k}}(\Omega)} \leqslant c \varepsilon^{-2 / q_{k}} \theta\left(\varepsilon, 1, p^{\prime}\right)\|g\|_{L_{p}(\Omega)}
$$

где $3<q_{1}<\infty, 2<q_{2}<\infty, 1<p<3,1 / p \leqslant 1 / q_{k}+1 / 2$; 


$$
\left\|\frac{\partial}{\partial x_{s}}\left(L_{\varepsilon} \partial_{j} g\right)_{k}\right\|_{L_{q}(\Omega)} \leqslant c\left\{(\theta(\varepsilon, 1, q)+\theta(\varepsilon, 2, q)) \theta\left(\varepsilon, 1, p^{\prime}\right)\|g\|_{L_{p}(\Omega)}+\|g\|_{L_{q}(\Omega)}\right\},
$$

где $3 / 2<q<\infty$, если $(k, s)=(1,2)$, и $1<q<\infty$, если $(k, s) \neq(1,2) ; 1<p<3$, $1 / p \leqslant 1 / q+1 / 2$.

Сравнивая оценки объемного потенциала с оценками (1.109), убеждаемся в том, что

$$
\left\|\left(R_{\varepsilon} f\right)_{k}\right\|_{L_{q_{k}}(\Omega)} \leqslant c \varepsilon^{-2+2\left(1 / p-1 / q_{k}\right)}\|f\|_{L_{p}(\Omega)}
$$

при $1<p \leqslant q_{k}<\infty, 1-1 / p+1 / q_{1} \in(0,1 / 3), 1-1 / p+1 / q_{2} \in(0,1 / 2)$, т.е. оценки разрешаюшего оператора полностью определяются оценками оператора объемного потенциала. Однако для производных этот факт не имеет места, и приходится отдельно использовать оценки для $U_{\varepsilon}$ и для $L_{\varepsilon}$.

\section{§2. Асимптотическое разложение}

Рассмотрим задачу обтекания для системы Навье-Стокса

$$
\begin{gathered}
\Delta u-\nabla p=2 \varepsilon(u, \nabla) u, \\
\operatorname{div} u=0,\left.\quad u\right|_{S}=b,\left.\quad u\right|_{\infty}=e^{1}
\end{gathered}
$$

во внешней области $D$ с границей $S$ класса $C^{\infty}$.

Осуществляя в системе Навье-Стокса (0.1) замену $u=v+e^{1}$, для определения $v$ получим систему

$$
\begin{gathered}
\Delta v-\nabla q-2 \varepsilon \frac{\partial}{\partial x_{1}} v=2 \varepsilon(v, \nabla) v \\
\operatorname{div} v=0,\left.\quad v\right|_{S}=b-e^{1},\left.\quad v\right|_{\infty}=0 .
\end{gathered}
$$

Будем искать решение системы $(2.2)$ при малых $\varepsilon$ в виде формального ряда ${ }^{1}$

$$
v=\sum_{k=0}^{\infty} \varepsilon^{k}\left(v_{\mathrm{o} k}+v_{\mathrm{s} k}\right) \text {. }
$$

Предполагая, что $v_{\mathrm{o} k}$ и $v_{\mathrm{S} k}$ при $k=0,1,2, \ldots$ удовлетворяют уравнениям

$$
\begin{gathered}
\Delta v_{\mathrm{O} k}-\nabla q_{\mathrm{O} k}-2 \varepsilon \frac{\partial}{\partial x_{1}} v_{\mathrm{O} k}=2 \varepsilon \sum_{m+n=k}\left(v_{\mathrm{o} m}, \nabla\right) v_{\mathrm{o} n}+2 \frac{\partial}{\partial x_{1}} v_{\mathrm{S} k-1} \\
+2 \sum_{m+n=k-1}\left\{\left(v_{\mathrm{s} m}+v_{\mathrm{o} m}, \nabla\right) v_{\mathrm{S} n}+\left(v_{\mathrm{s} m}, \nabla\right) v_{\mathrm{o} n}\right\} \\
\operatorname{div} v_{\mathrm{o} k}=0,\left.\quad v_{\mathrm{O} k}\right|_{\infty}=0 \\
\Delta v_{\mathrm{S} k}-\nabla q_{\mathrm{S} k}=0, \quad \operatorname{div} v_{\mathrm{S} k}=0,\left.\quad v_{\mathrm{S} k}\right|_{\infty}=0 \\
\left.\left(v_{\mathrm{S} k}+v_{\mathrm{o} k}\right)\right|_{S}=\left(b-e^{1}\right) \delta_{k 0}
\end{gathered}
$$

получаем, что формально ряд (2.3) удовлетворяет задаче (2.2).

\footnotetext{
${ }^{1}$ Поля $v_{\mathrm{O} k}$ и $v_{\mathrm{S} k}$ - решения соответственно системы Озеена (2.4) и системы Стокса $(2.5),(2.6)$.
} 
Отметим, что после подстановки

$$
v^{N}=\sum_{k=0}^{N} \varepsilon^{k}\left(v_{\mathrm{o} k}+v_{\mathrm{s} k}\right)
$$

в систему (2.2) получаем соотношения

$$
\begin{gathered}
\Delta v^{N}-\nabla q^{N}-2 \varepsilon \frac{\partial}{\partial x_{1}} v^{N}-2 \varepsilon\left(v^{N}, \nabla\right) v^{N}=2 \varepsilon^{N+1} \sum_{m+n=N}\left(v_{\mathrm{o} m}, \nabla\right) v_{\mathrm{o} n} \\
-2 \varepsilon^{N+1} \frac{\partial}{\partial x_{1}} v_{\mathrm{S} N}-2 \sum_{k=N}^{2 N} \varepsilon^{k+1} \sum_{\substack{m+n=k \\
m, n \leqslant N}}\left\{\left(v_{\mathrm{S} m}+v_{\mathrm{o} m}, \nabla\right)\left(v_{\mathrm{S} n}+v_{\mathrm{o} n}\right)\right\} \\
\operatorname{div} v^{N}=0,\left.\quad v^{N}\right|_{S}=b-e^{1},\left.\quad v^{N}\right|_{\infty}=0 .
\end{gathered}
$$

Наша дальнейшая цель - построить ряд (2.3), который является асимптотическим разложением решения задачи обтекания при $\varepsilon \rightarrow 0$.

2.1. Построение нулевого приближения. Полагая в (2.4) $k=0, y=\varepsilon x$, $v=v(y)=v_{\mathrm{o} 0}(y / \varepsilon)$, приходим к системе Озеена

$$
\begin{gathered}
\Delta v-\nabla q-2 \frac{\partial}{\partial y_{1}} v=2(v, \nabla) v, \\
\operatorname{div} v=0,\left.\quad v\right|_{\infty}=0,
\end{gathered}
$$

которую будем рассматривать в области $\mathbb{R}^{2} \backslash\{0\}$.

Обращая линейную часть, получим уравнение

$$
v=v^{1}+2 U[(v, \nabla) v]
$$

где $v^{1}$ - решение однородной линеаризованной системы Озеена в области $\mathbb{R}^{2} \backslash\{0\}$, $U$ - оператор объемного потенциала при $\varepsilon=1$. В качестве $v^{1}$ возьмем решение вида $v^{1}=\delta_{1} u^{1}+\delta_{2} u^{2}$, где $u^{k}$ - фундаментальное решение при $\varepsilon=1, \delta_{j}$ - произвольные постоянные.

Оператор $A v=U[(v, \nabla) v]$ в координатной форме имеет вид

$$
(A v)_{k}=\partial_{1} u_{1}^{k} *\left(v_{1}\right)^{2}+\left(\partial_{2} u_{1}^{k}+\partial_{1} u_{2}^{k}\right) *\left(v_{1} v_{2}\right)+\partial_{2} u_{2}^{k} *\left(v_{2}\right)^{2} .
$$

Заметим, что компоненты фундаментального решения различаются по поведению на бесконечности. Для учета этого факта при исследовании уравнения (2.10) введем пространство $L_{p_{1} p_{2}}\left(\mathbb{R}^{2}\right)$ векторных полей $u=\left(u_{1}, u_{2}\right)$ с нормой $\|u\|_{p_{1} p_{2}}=$ $\left\|u_{1}\right\|_{L_{p_{1}}\left(\mathbb{R}^{2}\right)}+\left\|u_{2}\right\|_{L_{p_{2}}\left(\mathbb{R}^{2}\right)}$. В частности, $v^{1}=\delta_{1} u^{1}+\delta_{2} u^{2} \in L_{p_{1} p_{2}}\left(\mathbb{R}^{2}\right)$ при $3<$ $p_{1}<\infty, 2<p_{2}<\infty$.

Теперь из оценок сверток (1.14) легко проверяется, что оператор $A$ непрерывен из $L_{p_{1} p_{2}}\left(\mathbb{R}^{2}\right)$ в $L_{q_{1} q_{2}}\left(\mathbb{R}^{2}\right)$ при вьполнении условий

$$
\begin{aligned}
1 \leqslant p_{1}, p_{2}, q_{1}, q_{2} \leqslant \infty, \quad m<1 / q_{1}<m_{1}, \quad m<1 / q_{2}<m_{2} \\
m=\max \left(2 / p_{1}, 2 / p_{2}, 1 / p_{1}+1 / p_{2}\right)-1 / 2<1, \\
m_{1}=\min \left(2 / p_{1}, 2 / p_{2}, 1 / p_{1}+1 / p_{2}-1 / 3\right) \\
m_{2}=\min \left(2 / p_{1}, 2 / p_{2}, 1 / p_{1}+1 / p_{2}\right)
\end{aligned}
$$


$\mathrm{B}$ частности, если $p_{2}<3<p_{1}$ и $p_{1}, p_{2}$ достаточно близки к трем, то можно положить $q_{1}=p_{1}, q_{2}=p_{2}$. Тогда, применяя теорему о неявных операторах (см., например, $[9$, с. 351$])$, получаем, что уравнение (2.10) имеет семейство решений $v=v(\delta, y)$, принадлежащих пространству $L_{p_{1} p_{2}}\left(\mathbb{R}^{2}\right)$, аналитически зависящих от параметров $\delta=\left(\delta_{1}, \delta_{2}\right)$ в некоторой области $|\delta|<\varrho$ и представимых в виде ряда:

$$
v(\delta)=\delta_{1} u^{1}+\delta_{2} u^{2}+\sum_{|\alpha| \geqslant 2} \delta^{\alpha} v_{\alpha}
$$

(здесь $\alpha=\left(\alpha_{1}, \alpha_{2}\right)$ является мультииндексом, $\left.\delta^{\alpha}=\delta_{1}^{\alpha_{1}} \delta_{2}^{\alpha_{2}}\right)$, причем $v_{\alpha}$ определяются рекуррентными формулами

$$
v_{\alpha}=2 U\left[\sum_{\beta+\gamma=\alpha}\left(v_{\beta}, \nabla\right) v_{\gamma}\right]
$$

где $v_{00}=0, v_{10}=u^{1}, v_{01}=u^{2}$. Из неравенств (2.12) методом "шнуровки" устанавливаем, что $v \in L_{q_{1} q_{2}}\left(\mathbb{R}^{2}\right)$ при $3<q_{1}<\infty, 2<q_{2}<\infty, A v \in L_{q_{1} q_{2}}\left(\mathbb{R}^{2}\right)$ при $2<q_{1} \leqslant \infty, 3 / 2<q_{2} \leqslant \infty$. Далее, используя оценку (1.14), получаем, что $\nabla A v \in L_{q}\left(\mathbb{R}^{2}\right)$ при $3 / 2<q<\infty$, поэтому $\nabla v \in L_{q}\left(\mathbb{R}^{2}\right)$ при $3 / 2<q<2$. Тогда $(v, \nabla) v \in L_{q}\left(\mathbb{R}^{2}\right)$ при $1<q<2$ и $\partial^{2} A v \in L_{q}\left(\mathbb{R}^{2}\right)$ при $1<q<2$.

Отметим также, что из результатов о внутренней регулярности системы Навье-Стокса вытекает, что полученное решение является бесконечно дифференцируемым в области $\mathbb{R}^{2} \backslash\{0\}$.

Таким образом, установлена

Лемма 2.1. Система Озеена (2.9) имеет семейство решений $v=v(\delta, y)$, бесконечно дифференцируемых в области $\mathbb{R}^{2} \backslash\{0\}$, аналитически зависящих от $\delta$ при $|\delta|<\varrho$, причем выполняются следующие условия:

$$
\begin{gathered}
v \in L_{q_{1} q_{2}}\left(\mathbb{R}^{2}\right), \quad 3<q_{1}<\infty, \quad 2<q_{2}<\infty ; \\
\nabla v \in L_{q}\left(\mathbb{R}^{2}\right), \quad 3 / 2<q<2 ; \\
\left(v-\delta_{1} u^{1}-\delta_{2} u^{2}\right) \in L_{q_{1} q_{2}}\left(\mathbb{R}^{2}\right) \cap C\left(\mathbb{R}^{2}\right), \quad 2<q_{1} \leqslant \infty, \quad 3 / 2<q_{2} \leqslant \infty ; \\
\nabla\left(v-\delta_{1} u^{1}-\delta_{2} u^{2}\right) \in L_{q}\left(\mathbb{R}^{2}\right), \quad 3 / 2<q \leqslant \infty ; \\
\partial^{2}\left(v-\delta_{1} u^{1}-\delta_{2} u^{2}\right) \in L_{q}\left(\mathbb{R}^{2}\right), \quad 1<q<2 ; \\
\left\|v-\delta_{1} u^{1}-\delta_{2} u^{2}\right\|_{C\left(\mathbb{R}^{2}\right)} \leqslant c|\delta|^{2} .
\end{gathered}
$$

Перейдем к построению поля $v_{\mathrm{S} 0}$. В качестве решения уравнения (2.4) возьмем $v_{\mathrm{o} 0}(x)=v(\delta, \varepsilon x)$. Для определения $v_{\mathrm{s} 0}$ имеем систему Стокса

$$
\begin{gathered}
\Delta v_{\mathrm{S} 0}-\nabla q_{\mathrm{S} 0}=0, \\
\operatorname{div} v_{\mathrm{S} 0}=0,\left.\quad v_{\mathrm{S} 0}\right|_{S}=a=b-e^{1}-\left.v_{\mathrm{o} 0}\right|_{S},\left.\quad v_{\mathrm{S} k}\right|_{\infty}=0 .
\end{gathered}
$$

Условия ее разрешимости

$$
\left(a, \psi^{j}\right)=0, \quad j=1,2,
$$

приводят к определению $\delta_{1}, \delta_{2}$ как функций от $\varepsilon$. Действительно, учитывая разложение фундаментального решения в ряд и аналитичность $v_{\mathrm{o} 0}-\delta_{1} u_{\varepsilon}^{1}-\delta_{2} u_{\varepsilon}^{2}$ как элемента из $C\left(\mathbb{R}^{2}\right)$, приведем систему $(2.16)$ к виду

$$
\beta_{j}+\frac{1}{4 \pi} \ln \frac{2}{\varepsilon} \delta_{i j} \delta_{i}+f_{j}(\delta, \varepsilon)=0, \quad j=1,2,
$$


где $\beta_{j}=\left(b-e^{1}, \psi^{j}\right), f_{j}(\delta, \varepsilon)$ аналитична по $\delta$ как элемент пространства $C\left[0, \varepsilon_{0}\right]$, причем $f_{j}(0, \varepsilon)=0$. Применяя теорему о неявной функции или прицип Банаха, убеждаемся, что при достаточно малом $\varepsilon_{0}>0$ существует единственное решение $\delta_{j}=\delta_{j}(\varepsilon)$ системы $(2.17)$, непрерывное по $\varepsilon$ на $\left[0, \varepsilon_{0}\right]$ и удовлетворяющее условиям

$$
\delta_{j}=-4 \pi \beta_{j}\left(\ln \frac{2}{\varepsilon}\right)^{-1}+O\left(\left(\ln \frac{1}{\varepsilon}\right)^{-2}\right) .
$$

В частности, при $b=0$

$$
\delta_{1}=4 \pi\left(\ln \frac{2}{\varepsilon}\right)^{-1}+O\left(\left(\ln \frac{1}{\varepsilon}\right)^{-2}\right), \quad \delta_{2}=O\left(\left(\ln \frac{1}{\varepsilon}\right)^{-2}\right) .
$$

При таком выборе $\delta_{j}$ выполнено условие разрешимости системы Стокса, гладкость граничных значений определяется гладкостью $b$ и при $b \in C^{\infty}(S)$ решение $v_{\mathrm{S} 0}$ бесконечно дифференцируемо вплоть до границы и удовлетворяет следующим оценкам на бесконечности:

$$
\left|\partial^{\alpha} v_{\mathrm{s} 0}(x)\right| \leqslant c|x|^{-|\alpha|-1} .
$$

Таким образом, установлен следующий результат.

Лемма 2.2. Существует $\varepsilon_{0}>0$ такое, что при $0 \leqslant \varepsilon \leqslant \varepsilon_{0}$ определено нулевое приближение $v^{0}(\varepsilon, x)=v_{\mathrm{o} 0}(\varepsilon, x)+v_{\mathrm{S} 0}(\varepsilon, x)\left(v_{\mathrm{o} 0}(\varepsilon, x)=v(\delta(\varepsilon), \varepsilon x)\right.$, где $v=v(\delta, x)$ определено в лемме 2.1), непрерывное по $\varepsilon$, бесконечно дифференцируемое вплоть до границь и удовлетворяющее следующим оценкам:

$-\partial \Omega я \tilde{v}_{\mathrm{o} 0}(\varepsilon, x)=v_{\mathrm{o} 0}\left(\varepsilon, \varepsilon^{-1} x\right)$

$$
\begin{gathered}
\left\|\tilde{v}_{\mathrm{O} 0}\right\|_{L_{q_{1} q_{2}}\left(\mathbb{R}^{2}\right)} \leqslant c\left(\ln \frac{1}{\varepsilon}\right)^{-1}, \quad 3<q_{1}<\infty, \quad 2<q_{2}<\infty ; \\
\left\|\nabla \tilde{v}_{\mathrm{O} 0}\right\|_{L_{q}\left(\mathbb{R}^{2}\right)} \leqslant c\left(\ln \frac{1}{\varepsilon}\right)^{-1}, \quad \frac{3}{2}<q<2 ; \\
\left\|\tilde{v}_{\mathrm{O} 0}-\delta_{1}(\varepsilon) u^{1}-\delta_{2}(\varepsilon) u^{2}\right\|_{L_{q_{1} q_{2}}\left(\mathbb{R}^{2}\right) \cap C\left(\mathbb{R}^{2}\right)} \leqslant c\left(\ln \frac{1}{\varepsilon}\right)^{-2}, \\
2<q_{1} \leqslant \infty, \quad \frac{3}{2}<q_{2} \leqslant \infty ; \\
\left\|\nabla\left(\tilde{v}_{\mathrm{O} 0}-\delta_{1}(\varepsilon) u^{1}-\delta_{2}(\varepsilon) u^{2}\right)\right\|_{L_{q}\left(\mathbb{R}^{2}\right)} \leqslant c\left(\ln \frac{1}{\varepsilon}\right)^{-2}, \quad \frac{3}{2}<q \leqslant \infty ; \\
\left\|\partial^{2}\left(\tilde{v}_{\mathrm{O} 0}-\delta_{1}(\varepsilon) u^{1}-\delta_{2}(\varepsilon) u^{2}\right)\right\|_{L_{q}\left(\mathbb{R}^{2}\right)} \leqslant c\left(\ln \frac{1}{\varepsilon}\right)^{-2}, \quad 1<q \leqslant 2 ; \\
- \text { для } v_{\mathrm{S} 0}\left|\partial^{\alpha} v_{\mathrm{S} 0}(x)\right| \leqslant c(1+|x|)^{-|\alpha|-1} .
\end{gathered}
$$

2.2. Высшие приближения. Основным построениям предпошлем ряд лемм.

Лемма 2.3. Оператор $A: L_{p_{1} p_{2}}\left(\mathbb{R}^{2}\right) \rightarrow L_{q_{1} q_{2}}\left(\mathbb{R}^{2}\right)$, определяемый фор.мулой

$$
A v=U\left[\left(\nabla, \tilde{v}_{\mathrm{O} 0}\right) v+(\nabla, v) \tilde{v}_{\mathrm{o} 0}\right] \text {, }
$$


ограничен при выполнении условий

$$
\begin{aligned}
& \max \left(1 / p_{1}, 1 / p_{2}\right)-1 / 2<1 / q_{1}<\min \left(2 / 3,1 / p_{2}, 1 / p_{1}+1 / 6\right), \\
& \max \left(1 / p_{1}, 1 / p_{2}\right)-1 / 2<1 / q_{2}<\min \left(1,1 / p_{1}+1 / 3,1 / p_{2}+1 / 3\right) .
\end{aligned}
$$

При этом

$$
\|A\|_{L_{p_{1} p_{2}} \rightarrow L_{q_{1} q_{2}}} \leqslant c\left(\ln \frac{1}{\varepsilon}\right)^{-1},
$$

где с зависит только от $p_{1}, p_{2}, q_{1}, q_{2}$

В частности, оператор А ограничен, если только

$$
1 / p_{2}-1 / 2<1 / p_{1}<1 / p_{2}<1 / p_{1}+1 / 3<1 .
$$

Доказательство легко следует из оценок сверток (1.14) и неравенства (2.21).

Лемма 2.4. Существует $\varepsilon_{0}>0$ такое, что при $0<\varepsilon \leqslant \varepsilon_{0}$ возмущенная система Озеена

$$
\begin{gathered}
\Delta v-\nabla p-2 \frac{\partial}{\partial y_{1}} v-2\left[\left(\nabla, \tilde{v}_{\mathrm{o} 0}\right) v+(\nabla, v) \tilde{v}_{\mathrm{o} 0}\right]=f, \\
\operatorname{div} v=0
\end{gathered}
$$

рассматриваемая в $\mathbb{R}^{2}$ при $f \in L_{p}\left(\mathbb{R}^{2}\right)$ для всех $p \in(1,2)$, имеет решение, удовлетворяющее следующим условиям:

$$
\begin{aligned}
v & \in L_{q_{1} q_{2}}\left(\mathbb{R}^{2}\right) & & \forall q_{1} \in(3, \infty), \quad \forall q_{2} \in(2, \infty) ; \\
\nabla v & \in L_{q}\left(\mathbb{R}^{2}\right) & & \forall q \in(3 / 2, \infty) ; \\
\partial^{2} v & \in L_{q}\left(\mathbb{R}^{2}\right) & & \forall q \in(1,2) ;
\end{aligned}
$$

причем

$$
\|v\|_{q_{1} q_{2}} \leqslant c\|f\|_{r}
$$

для любого $r \in(1,2)$, удовлетворяющего условию

$$
\begin{gathered}
\max \left(1 / q_{1}+2 / 3,1 / q_{2}+1 / 2,5 / 6\right)<1 / r<1 \\
\|\nabla v\|_{q} \leqslant c\left(\|f\|_{r}+\|f\|_{s}\right)
\end{gathered}
$$

для любьх $s, r \in(1,2)$, удовлетворяющих условиям

$$
\begin{gathered}
1 / 3+1 / q<1 / s<1 / 2+1 / q, \\
\max (1 / q-1 / \varrho+2 / 3,5 / 6)<1 / r<1,
\end{gathered}
$$

где உ можнно выбрать любым из интервала $(\max (3, q), \infty)$.

ДокАЗАТЕЛЬСтво. Обрашая линеаризованный оператор Озеена, приходим к уравнению

$$
v-A v=U f
$$

где $A$ - оператор (2.27).

Из оценок объемного потенциала (1.14) следует, что $U f$ удовлетворяет условиям (2.32)-(2.34), причем выполняются соотношения $(2.35)-(2.39)$, в которых $v$ следует заменить на $U f$. 
Возьмем $p_{1}=6, p_{2}=3$. Из леммы 2.3 вытекает, что оператор $A$ ограничен в $L_{6,3}\left(\mathbb{R}^{2}\right)$ и имеет малую норму при достаточно малом $\varepsilon: 0<\varepsilon \leqslant \varepsilon_{0}$. Поэтому уравнение (2.40) имеет единственное решение в этом пространстве, причем

$$
\|v\|_{6,3} \leqslant c\|U f\|_{6,3} \leqslant c\|f\|_{r}
$$

при любом $r \in(1,6 / 5)$.

Анализируя условия (2.28), получим

$$
\|A v\|_{q_{1} q_{2}} \leqslant c\|v\|_{6,3} \quad \forall q_{1} \in(3, \infty), \quad \forall q_{2} \in(2, \infty) .
$$

Из неравенств (2.35), (2.36), где $v$ заменено на $U f$, и (2.41) вытекает справедливость оценок $(2.32),(2.35),(2.36)$, так как из уравнения (2.40) следует, что

$$
\|v\|_{q_{1} q_{2}} \leqslant\|A v\|_{q_{1} q_{2}}+\|U f\|_{q_{1} q_{2}} .
$$

Далее, $\|\nabla A v\|_{q} \leqslant c\left\|\tilde{v}_{\text {о } 0}\right\|_{\varrho}\|v\|_{m}$ при $1 / q=1 / \varrho+1 / m, \varrho, m \in(3, \infty)$. Но из (2.35), (2.36) при $q_{1}=q_{2}=m$ следует, что $\|v\|_{m} \leqslant c\|f\|_{r}$ при любом $r$, удовлетворяющем условию (2.39). Остается заметить, что

$$
\|\nabla v\|_{q} \leqslant c\left(\|\nabla U f\|_{q}+\|v\|_{m}\right) \leqslant c\left(\|f\|_{s}+\|f\|_{r}\right),
$$

где $s$ удовлетворяет условию (2.38).

Наконец,

$$
\left\|\left(\tilde{v}_{\mathrm{o} 0}, \nabla\right) v+(v, \nabla) \tilde{v}_{\mathrm{o} 0}\right\|_{q} \leqslant c\left(\left\|\tilde{v}_{\mathrm{o} 0}\right\|_{\varrho}\|\nabla v\|_{l}+\|v\|_{\varrho}\left\|\nabla \tilde{v}_{\mathrm{o} 0}\right\|_{l}\right),
$$

где $1 / q=1 / \varrho+1 / l, \varrho \in(3, \infty), l \in(3 / 2, \infty)$, что и влечет $(2.34)$ ввиду ограниченности оператора $\partial^{2} U$ в любом пространстве $L_{q}\left(\mathbb{R}^{2}\right)$ при $q \in(1, \infty)$.

Лемма 2.5. Существует $\varepsilon_{0}>0$ mакое, ито при $0<\varepsilon \leqslant \varepsilon_{0}$ однородная возмущенная система Озеена

$$
\begin{gathered}
\Delta v-\nabla p-2 \frac{\partial}{\partial y_{1}} v-2\left[\left(\nabla, \tilde{v}_{\mathrm{o} 0}\right) v+(\nabla, v) \tilde{v}_{\mathrm{o} 0}\right]=0, \\
\operatorname{div} v=0
\end{gathered}
$$

рассматриваемая в $\mathbb{R}^{2} \backslash\{0\}$, имеет решение вида

$$
v=\delta_{1}\left(u^{1}+w^{1}\right)+\delta_{2}\left(u^{2}+w^{2}\right),
$$

где $\delta_{j}$ - произвольные постоянные, $u^{j}$ - фундаментальное решение при $\varepsilon=1$, $w^{j}-$ поля, удовлетворяющие условиям:

$$
\begin{gathered}
\left\|w^{j}\right\|_{p_{1} p_{2}} \leqslant c\left(\ln \frac{1}{\varepsilon}\right)^{-1} \quad \forall p_{1} \in(3, \infty), \quad \forall p_{2} \in(2, \infty) ; \\
\left\|\nabla w^{j}\right\|_{p} \leqslant c\left(\ln \frac{1}{\varepsilon}\right)^{-1} \quad \forall p \in\left(\frac{3}{2}, \infty\right) ; \\
\left\|w^{j}\right\|_{C\left(\mathbb{R}^{2}\right)} \leqslant c\left(\ln \frac{1}{\varepsilon}\right)^{-1} ; \\
\left\|\partial^{2} w^{j}\right\|_{p} \leqslant c\left(\ln \frac{1}{\varepsilon}\right)^{-1} \quad \forall p \in(1,2) .
\end{gathered}
$$


ДокАЗАТЕЛЬСТво. Полагая $v=u^{j}+w^{j}$, для определения $w^{j}$ имеем систему из леммы 2.4 , в которой $f=2\left[\left(\nabla, \tilde{v}_{\mathrm{o} 0}\right) u^{j}+\left(\nabla, u^{j}\right) \tilde{v}_{\mathrm{o} 0}\right]$. Учитывая, что

$$
\|f\|_{p} \leqslant c\left(\left\|\tilde{v}_{\mathrm{o} 0}\right\|\left\|_{r}\right\| \nabla u^{j}\left\|_{s}+\right\| u^{j}\left\|_{r}\right\| \nabla \tilde{v}_{\mathrm{o} 0} \|_{s}\right)
$$

при $1 / p=1 / r+1 / s, r \in(3, \infty), s \in(3 / 2, \infty),\left\|\tilde{v}_{\text {o } 0}\right\|_{r} \leqslant c(\ln 1 / \varepsilon)^{-1},\left\|\nabla \tilde{v}_{\mathrm{o} 0}\right\|_{s} \leqslant$ $c(\ln 1 / \varepsilon)^{-1}$, получаем, что $f \in L_{p}\left(\mathbb{R}^{2}\right) \forall p \in(1,2)$ и $\|f\|_{p} \leqslant c(\ln 1 / \varepsilon)^{-1}$.

Поэтому оценки для $w^{j}$ вытекают из леммы 2.4 , а непрерьвность - из теоремы вложения Соболева.

Tеорема 2.1. Существует такое $\varepsilon_{N}$, ито при $0<\varepsilon \leqslant \varepsilon_{N}$ определено асимптотическое разложение $v^{N}$ вида (2.7), удовлетворяющее следующим условиям:

1) для любого сколь угодно малого $\eta>0$ справедливы оченки:

$$
\begin{aligned}
\left\|v_{\mathrm{s} k}\right\|_{L_{q}(D)} \leqslant c_{\eta} \varepsilon^{-\eta} & \forall q \in(2, \infty], \\
\left\|\nabla v_{\mathrm{s} k}\right\|_{L_{q}(D)} \leqslant c_{\eta} \varepsilon^{-\eta} & \forall q \in(1, \infty]
\end{aligned}
$$

для полей $v_{\mathrm{s} k}(\varepsilon, x)$;

$$
\begin{aligned}
& \left\|\left(\tilde{v}_{\mathrm{O} k}\right)_{j}\right\|_{L_{q_{j}}\left(\mathbb{R}^{2}\right)} \leqslant c_{\eta} \varepsilon^{-\eta} \quad \forall q_{1} \in(3, \infty), \quad \forall q_{2} \in(2, \infty), \\
& \left\|\partial_{j} \tilde{v}_{\mathrm{o} k}\right\|_{L_{q_{j}}\left(\mathbb{R}^{2}\right)} \leqslant c_{\eta} \varepsilon^{-\eta} \quad \forall q_{1} \in(1,2), \quad \forall q_{2} \in\left(\frac{3}{2}, 2\right)
\end{aligned}
$$

для полей $\tilde{\mathrm{o}}_{\mathrm{o}}(\varepsilon, y)=v_{\mathrm{o} k}(\varepsilon, y / \varepsilon)$

2) существуют функщии $\delta_{j k}=\delta_{j k}(\varepsilon)$ такие, что

$$
\left\|\nabla\left(\tilde{v}_{\mathrm{o} k}-\sum_{j=1}^{2} \delta_{j k}(\varepsilon) u^{j}\right)\right\|_{q} \leqslant c_{\eta} \varepsilon^{-\eta} \quad \forall q \in(2, \infty)
$$

(функции $\delta_{j k}(\varepsilon)$ определяются в прочессе доказательства).

ДокАЗАТЕльСтво. Предположим, что поля $v_{\mathrm{s} k}, v_{\mathrm{o} k}, k=1,2, \ldots, N-1$, обладаюшие нужными свойствами, уже построены. Для определения $v_{\mathrm{o}} N$ имеем систему

$$
\begin{gathered}
\Delta v_{\mathrm{O} N}-\nabla q_{\mathrm{o} N}-2 \varepsilon \frac{\partial}{\partial x_{1}} v_{\mathrm{o} N}-2 \varepsilon\left[\left(\nabla, v_{\mathrm{o} 0}\right) v_{\mathrm{o} N}+\left(\nabla, v_{\mathrm{o} N}\right) v_{\mathrm{o} 0}\right]=F, \\
\operatorname{div} v_{\mathrm{o} N}=0, \\
F=2 \varepsilon \sum_{m+n=N ; m, n \neq 0, N}\left(v_{\mathrm{o} m}, \nabla\right) v_{\mathrm{O} n}+2 \frac{\partial}{\partial x_{1}} v_{\mathrm{s}, N-1}\left\{v_{m+n=N-1}\left\{\left(v_{\mathrm{s} m}+v_{\mathrm{o} m}, \nabla\right) v_{\mathrm{S} n}+\left(v_{\mathrm{S} m}, \nabla\right) v_{\mathrm{o} n}\right\} .\right. \\
+2 \sum_{m+1}
\end{gathered}
$$

Осушествляя замену переменных $y=\varepsilon x$ и используя для произвольной функции $g$ обозначение $\tilde{g}(y)=g(y / \varepsilon)$, для $\tilde{v}_{\text {о } N}$ получаем систему

$$
\begin{gathered}
\Delta \tilde{v}_{\mathrm{O} N}-\varepsilon^{-1} \nabla \tilde{q}_{\mathrm{O} N}-2 \frac{\partial}{\partial y_{1}} \tilde{v}_{\mathrm{O} N}-2\left[\left(\nabla, \tilde{v}_{\mathrm{O} 0}\right) \tilde{v}_{\mathrm{O} N}+\left(\nabla, \tilde{v}_{\mathrm{O} N}\right) \tilde{v}_{\mathrm{O} 0}\right]=\varepsilon^{-2} \widetilde{F} \\
\operatorname{div} v_{\mathrm{O} N}=0
\end{gathered}
$$


Продолжив $\tilde{v}_{\mathrm{S}}, \frac{\partial}{\partial y_{1}} \tilde{v}_{\mathrm{s}}$ нулем на $\mathbb{R}^{2} \backslash\{\varepsilon D\}$, будем рассматривать эту систему на $\mathbb{R}^{2} \backslash\{0\}$. Для $\varepsilon^{-2} \widetilde{F}$ имеем оценку

$$
\begin{aligned}
\left\|\varepsilon^{-2} \widetilde{F}\right\|_{p}= & \varepsilon^{2 / p-2}\|F\|_{L_{p}(D)} \leqslant \varepsilon^{2 / p-2}\left\{2\left\|\frac{\partial}{\partial x_{1}} v_{\mathrm{S}, N-1}\right\|_{p}\right. \\
& +2 \sum_{m+n=N-1}\left\{\left\|v_{\mathrm{S} n}\right\|_{p_{1}}\left\|\nabla v_{\mathrm{S} m}\right\|_{p_{2}}+\varepsilon^{-2 / s_{1}}\left\|\tilde{v}_{\mathrm{o} n}\right\|_{\mathrm{S}_{1}}\left\|\nabla v_{\mathrm{S} m}\right\|_{\mathrm{S}_{2}}\right. \\
& \left.+\varepsilon^{1-2 / r_{2}}\left\|v_{\mathrm{S} n}\right\|_{r_{1}}\left\|\nabla \tilde{v}_{\mathrm{o} m}\right\|_{r_{2}}\right\} \\
& \left.+2 \varepsilon^{2-2 / q_{1}-2 / q_{2}} \sum_{m+n=N ; n, m \neq 0, N}\left\|\tilde{v}_{\mathrm{o} N}\right\|_{q_{1}}\left\|\nabla \tilde{v}_{\mathrm{o} m}\right\|_{q_{2}}\right\},
\end{aligned}
$$

где

$$
\begin{gathered}
1 / p=1 / p_{1}+1 / p_{2}=1 / s_{1}+1 / s_{2}=1 / r_{1}+1 / r_{2}=1 / q_{1}+1 / q_{2}, \\
p_{1}, r_{1} \in(2, \infty), \quad p_{2}, s_{2} \in(1, \infty), \quad s_{1}, q_{1} \in(3, \infty), \quad r_{2}, q_{2} \in(3 / 2,2) .
\end{gathered}
$$

Нас интересует случай $p \in(1,2)$. Но тогда $s_{1}$ и $r_{2}$ можно выбрать сколь угодно близкими к $\infty$ и 2 , так что можно считать, что $2 / s_{1}<\eta$ и $2 / r_{2}-1<\eta$. Поэтому верно неравенство

$$
\left\|\varepsilon^{-2} \widetilde{F}\right\|_{p} \leqslant c_{\eta} \varepsilon^{2 / p-2-2 \eta} .
$$

Применив теперь к системе (2.54) леммы $2.4,2.5$, установим, что сушествует решение $\tilde{v}_{\text {о } N}$, имеющее вид

$$
\tilde{v}_{\mathrm{o} N}=\delta_{1}\left(u^{1}+\widetilde{w}^{1}\right)+\delta_{2}\left(u^{2}+\widetilde{w}^{2}\right)+\tilde{v}
$$

где $\widetilde{w}^{j}$ удовлетворяют оценкам (2.44)-(2.47), $\tilde{v}$ удовлетворяет включениям (2.32)(2.34), а из оценок $(2.35),(2.37)$ с учетом (2.55) вытекает, что

$$
\|\tilde{v}\|_{q_{1} q_{2}} \leqslant c \varepsilon^{2 / r-2-3 \eta}
$$

где $q_{1}, q_{2}, r$ связаны соотношениями (2.36),

$$
\|\nabla \tilde{v}\|_{q} \leqslant c\left(\varepsilon^{2 / r-2-3 \eta}+\varepsilon^{2 / s-2-3 \eta}\right),
$$

где $s, r, q$ связаны соотношениями (2.38), (2.39).

Для оценки $\|\tilde{v}\|_{q_{1} q_{2}}$ можно выбрать $r$ сколь угодно близким к 1 , следовательно,

$$
\|\tilde{v}\|_{q_{1} q_{2}} \leqslant c \varepsilon^{-4 \eta} .
$$

Для оценки $\|\nabla \tilde{v}\|_{q}$ также можно полагать, что $2-2 / r<\eta$, а $2-2 / s<\eta$ при $q \in(3 / 2,2]$ и $2 / s-2>2 / q-1-\eta$ при $q \in(2, \infty)$. Таким образом, приходим к оценке

$$
\|\nabla \tilde{v}\|_{q} \leqslant \begin{cases}\varepsilon^{-4 \eta}, & q \in(3 / 2,2] \\ \varepsilon^{2 / q-1-4 \eta}, & q \in(2, \infty) .\end{cases}
$$

В частности, по вложениям Соболева пространство $W_{q}^{1}\left(\mathbb{R}^{2}\right)$ при $q>2$ непрерывно вложено в пространство $C\left(\mathbb{R}^{2}\right)$, следовательно,

$$
\|\tilde{v}\|_{C\left(\mathbb{R}^{2}\right)} \leqslant c \varepsilon^{-5 \eta} .
$$


Таким образом, $v_{\mathrm{o}} N$ обладает нужными свойствами.

Для определения $v_{\mathrm{s} N}$ имеем систему Стокса (2.5) с граничным условием (2.6). Ее условия разрешимости

$$
\left(\delta_{1}\left(u_{\varepsilon}^{1}+w_{1}\right)+\delta_{2}\left(u_{\varepsilon}^{2}+w_{2}\right), \psi^{j}\right)+\left(v, \psi^{j}\right)=0, \quad j=1,2,
$$

служат для определения функций $\delta_{j N}(\varepsilon)$. Ввиду соотношений

$$
\left(u_{\varepsilon}^{k}+w^{k}, \psi^{j}\right)=\delta_{k j} 4 \pi \ln \frac{2}{\varepsilon}+O(1)
$$

при достаточно малом $\varepsilon$ функции $\delta_{j N}=\delta_{j N}(\varepsilon)$ определяются однозначно и

$$
\left|\delta_{j N}(\varepsilon)\right| \leqslant c_{\eta} \varepsilon^{-4 \eta} .
$$

Этим завершается доказательство теоремы.

2.3. Обоснование асимптотики. Ищем решение задачи обтекания в виде

$$
v=v^{N}+\varepsilon^{N+1} w .
$$

Тогда для определения $w$ получаем систему

$$
\begin{gathered}
\Delta w-\nabla q-2 \varepsilon \frac{\partial}{\partial x_{1}} w-2 \varepsilon\left[\left(\nabla, v^{N}\right) w+(\nabla, w) v^{N}\right]-2 \varepsilon^{N+2}(w, \nabla) w=F, \\
\operatorname{div} w=0,\left.\quad w\right|_{\partial D}=0,\left.\quad w\right|_{\infty}=0, \\
F=2 \sum_{k=N+1}^{2 N} \varepsilon^{k-N} \sum_{m+n=k}\left(v_{\mathrm{s} m}+v_{\mathrm{o} m}, \nabla\right)\left(v_{\mathrm{s} n}+v_{\mathrm{o} n}\right) \\
+2 \frac{\partial}{\partial x_{1}} v_{\mathrm{S} N}+2 \sum_{m+n=N}\left\{\left(v_{\mathrm{s} m}+v_{\mathrm{o} m}, \nabla\right) v_{\mathrm{s} n}+\left(v_{\mathrm{s} m}, \nabla\right) v_{\mathrm{o} n}\right\} .
\end{gathered}
$$

Применяя разрешающий оператор для системы Озеена, приходим к следуюшему уравнению:

$$
w=R_{\varepsilon}\left\{2 \varepsilon\left[\left(v^{N}, \nabla\right) w+(w, \nabla) v^{N}\right]+2 \varepsilon^{N+2}(w, \nabla) w+F\right\} .
$$

ЛЕмма 2.6. Для любой точки области

$$
Q=\left\{\left(q_{1}, q_{2}\right) ; 1 / 6<1 / q_{1}<1 / q_{2}<1 / 2\right\}
$$

существует $\varepsilon_{0}\left(q_{1}, q_{2}\right)>0$ такое, что оператор $I-\mathscr{A}_{\varepsilon}$,

$$
\mathscr{A}_{\varepsilon} w=2 \varepsilon R_{\varepsilon}\left[\left(v^{N}, \nabla\right) w+(w, \nabla) v^{N}\right],
$$

обратим при $0<\varepsilon<\varepsilon_{0}\left(q_{1}, q_{2}\right)$ в пространстве $L_{q_{1} q_{2}}(D)$, причем

$$
\left\|\left(I-\mathscr{A}_{\varepsilon}\right)^{-1}\right\|_{L_{q_{1} q_{2}} \rightarrow L_{q_{1} q_{2}}} \leqslant 2 \varepsilon^{-2\left(1 / q_{2}-1 / q_{1}\right)} .
$$


ДокаЗАтельСтво. Рассмотрим уравнение

$$
\left(I-\mathscr{A}_{\varepsilon}\right) w=f .
$$

Применяя оператор растяжения $\left(T_{\varepsilon} u\right)(x)=u(\varepsilon x)$ и обозначая $\tilde{u}=T_{\varepsilon}^{-1} u$, приведем уравнение (2.62) к виду

$$
\widetilde{w}-T_{\varepsilon}^{-1} \mathscr{A}_{\varepsilon} T_{\varepsilon} \widetilde{w}=\tilde{f}
$$

Положим

$$
T_{\varepsilon}^{-1} \mathscr{A}_{\varepsilon} T_{\varepsilon} \widetilde{w}=\widetilde{w}^{1}+\widetilde{w}^{2},
$$

где

$$
\begin{aligned}
\widetilde{w}^{1} & =2 U\left[\left(\tilde{v}^{N}, \nabla\right) \widetilde{w}+(\tilde{w}, \nabla) \tilde{v}^{N}\right], \\
\widetilde{w}^{2} & =2 \varepsilon T_{\varepsilon}^{-1} L_{\varepsilon}\left[\left(v^{n}, \nabla\right) w+(w, \nabla) v^{N}\right] .
\end{aligned}
$$

Получим несколько более обшие оценки, чем это требуется для доказательства. Они будут использованы в дальнейшем. Заметим, что при рассмотрении $\widetilde{w}^{1}$ можно воспользоваться неравенством (2.29), так как для $\tilde{v}^{N}$ справедливы те же оценки, что и для $\tilde{v}_{\text {о } 0}$. Поэтому

$$
\left\|\widetilde{w}^{1}\right\|_{L_{q_{1} q_{2}}(\varepsilon D)} \leqslant c\left(\ln \varepsilon^{-1}\right)^{-1}\|\widetilde{w}\|_{L_{p_{1} p_{2}}(\varepsilon D)},
$$

где $\left(q_{1}, q_{2}\right)$ и $\left(p_{1}, p_{2}\right)$ связаны соотношениями $(2.28)$. В частности, можно положить $p_{1}=q_{1}, p_{2}=q_{2}$, если только вьполнены неравенства

$$
1 / q_{2}-1 / 2<1 / q_{1}<1 / q_{2}<1 / q_{1}+1 / 3<1
$$

Далее, применяя оценки (1.112) для оператора $L_{\varepsilon} \nabla$, имеем

$$
\begin{gathered}
\left\|\widetilde{w}^{2}\right\|_{L_{q_{1} q_{2}}(\varepsilon D)} \leqslant c \varepsilon\left(\ln \varepsilon^{-1}\right)^{1 / 2}\left(\left\|w_{1} v^{N}\right\|_{L_{2}(D)}+\left\|w_{2} v^{N}\right\|_{L_{2}(D)}\right) \\
\leqslant c\left(\ln \varepsilon^{-1}\right)^{1 / 2}\left(\left\|\widetilde{w}_{1} \tilde{v}^{N}\right\|_{L_{2}(\varepsilon D)}+\left\|\widetilde{w}_{2} \tilde{v}^{N}\right\|_{L_{2}(\varepsilon D)}\right) \\
\leqslant c\left(\ln \varepsilon^{-1}\right)^{1 / 2}\left(\left\|\widetilde{w}_{1}\right\|_{L_{p_{1}}(\varepsilon D)}\left\|\tilde{v}^{N}\right\|_{L_{s_{1}}(\varepsilon D)}\right. \\
\left.\quad+\left\|\widetilde{w}_{2}\right\|_{L_{p_{2}}(\varepsilon D)}\left\|\tilde{v}^{N}\right\|_{L_{s_{2}}(\varepsilon D)}\right),
\end{gathered}
$$

где

$$
\begin{gathered}
3<q_{1}, p_{1}, s_{1}, s_{2}<\infty, \quad 2<q_{2}, p_{2}<\infty \\
1 / p_{1}+1 / s_{1}=1 / p_{2}+1 / s_{2}=1 / 2 .
\end{gathered}
$$

С учетом того, что $\left\|\tilde{v}^{N}\right\|_{L_{s}\left(\mathbb{R}^{2}\right)} \leqslant\left(\ln \varepsilon^{-1}\right)^{-1}$ при $s>3$, получаем

$$
\left\|\widetilde{w}^{2}\right\|_{L_{q_{1} q_{2}}(\varepsilon D)} \leqslant c\left(\ln \varepsilon^{-1}\right)^{-1 / 2}\|\widetilde{w}\|_{L_{p_{1} p_{2}}(\varepsilon D)},
$$

где $q_{1}, q_{2}, p_{1}, p_{2}$ - любые числа, для которых выполняются условия (2.69). В частности, можно положить $p_{1}=q_{1}, p_{2}=q_{2}$, если только $q_{1} \in(3,6), q_{2} \in(2,6)$. Сравнивая оценки для $\widetilde{w}^{1}$ и $\widetilde{w}^{2}$, имеем

$$
\left\|T_{\varepsilon}^{-1} \mathscr{A}_{\varepsilon} T_{\varepsilon} \tilde{w}\right\|_{L_{q_{1} q_{2}}(\varepsilon D)} \leqslant c\left(\ln \varepsilon^{-1}\right)^{-1 / 2}\|\widetilde{w}\|_{L_{q_{1} q_{2}}(\varepsilon D)}
$$


при $\left(q_{1}, q_{2}\right) \in Q$.

Поскольку постоянная $c$ в оценке (2.71) зависит только от $\left(q_{1}, q_{2}\right)$, то существует такое $\varepsilon_{0}\left(q_{1}, q_{2}\right)>0$, что оператор $I-T_{\varepsilon}^{-1} \mathscr{A}_{\varepsilon} T_{\varepsilon}$ обратим в $L_{q_{1} q_{2}}(\varepsilon D)$ и

$$
\left\|\left(I-T_{\varepsilon}^{-1} \mathscr{A}_{\varepsilon} T_{\varepsilon}\right)^{-1}\right\|<2 .
$$

Но тогда оператор $I-\mathscr{A}_{\varepsilon}$ обратим в пространстве $L_{q_{1} q_{2}}(D)$ и справедлива оценка (2.61).

Обрашая оператор $I-\mathscr{A}_{\varepsilon}$, сведем уравнение $(2.62)$ к виду

$$
w=\mathscr{B}_{\varepsilon} w+g,
$$

где

$$
\begin{aligned}
\mathscr{B}_{\varepsilon} w & =2\left(I-\mathscr{A}_{\varepsilon}\right)^{-1} R_{\varepsilon} \varepsilon^{N+2}(w, \nabla) w, \\
g & =\left(I-\mathscr{A}_{\varepsilon}\right)^{-1} R_{\varepsilon} F .
\end{aligned}
$$

Из оценок (1.114) оператора $R_{\varepsilon}$ имеем

$$
\left\|\left(R_{\varepsilon} F\right)_{k}\right\|_{L_{q_{k}}(D)} \leqslant \varepsilon^{-2+2\left(1 / p-1 / q_{k}\right)}\|F\|_{L_{p}(D)},
$$

что с учетом неравенства (см. (2.55))

$$
\|F\|_{L_{p}(D)} \leqslant c_{\eta} \varepsilon^{-\eta}, \quad p \in(1,2)
$$

и того, что $p$ можно взять сколь угодно близким к 1 , приводит к оценке

$$
\left\|\left(R_{\varepsilon} F\right)_{k}\right\|_{L_{q_{k}}(D)} \leqslant c \varepsilon^{-2 / q_{k}-\eta}, \quad 3<q_{1}<\infty, \quad 2<q_{2}<\infty .
$$

Но тогда из (2.61) вытекает неравенство

$$
\left\|g_{k}\right\|_{L_{q_{k}}(D)} \leqslant c_{\eta} \varepsilon^{-2 / q_{k}-2\left(1 / q_{2}-1 / q_{1}\right)-2 \eta}
$$

если $1 / 6<1 / q_{1}<1 / q_{2}<1 / 2-\eta, 1 / q_{1} \leqslant 1 / 3-\eta$.

Теперь рассмотрим поле $R_{\varepsilon}[(\nabla, w) w]$ и представим его в виде суммы $\zeta^{1}+\zeta^{2}$, причем

$$
\begin{aligned}
& \zeta^{1}=2 U_{\varepsilon}[(\nabla, w) w] \\
& \zeta^{2}=2 L_{\varepsilon}[(\nabla, w) w] .
\end{aligned}
$$

Из условий непрерывности оператора $v \rightarrow U(\nabla, v) v$ (см. (2.12)) и оценок (1.14), (1.112) вытекает, что

$$
\left\|\zeta^{j}\right\|_{L_{q_{1} q_{2}(D)}} \leqslant c(\varepsilon)\|w\|_{L_{p_{1} p_{2}(D)}^{2}}^{2}
$$

где рост $c(\varepsilon)$ не превосходит $\varepsilon^{-2}$, а $p_{1}, p_{2}, q_{1}, q_{2}$ связаны соотношениями $(2.12)$ и соотношениями $2<p_{1}, p_{2}<6,3<q_{1}<\infty, 2<q_{2}<\infty$.

Теперь заметим, что оценки (2.61), (2.78), (2.81) заведомо справедливы, если положить $1 / q_{1}=1 / p_{1}=1 / 3-\eta, 1 / q_{2}=1 / p_{2}=1 / 3+\eta$. 
В результате получаем

$$
\begin{gathered}
\left\|\mathscr{B}_{\varepsilon} w\right\|_{L_{q_{1} q_{2}}(D)} \leqslant c \varepsilon^{N}\|w\|_{L_{q_{1} q_{2}}(D)}, \\
\left\|\mathscr{B}_{\varepsilon} w^{1}-\mathscr{B}_{\varepsilon} w^{2}\right\|_{L_{q_{1} q_{2}}(D)} \leqslant c \varepsilon^{N} \max _{j}\left\|w^{j}\right\|_{L_{q_{1} q_{2}}(D)}\left\|w^{1}-w^{2}\right\|_{L_{q_{1} q_{2}}(D)} \\
\left\|g_{k}\right\|_{L_{q_{k}}(D)} \leqslant c_{\eta} \varepsilon^{-2 / 3-8 \eta}
\end{gathered}
$$

Применяя принцип Банаха, устанавливаем,что уравнение (2.73) при достаточно малом $\varepsilon, 0<\varepsilon<\varepsilon_{0}$, имеет в некотором шаре пространства $L_{q_{1} q_{2}}(D)$ единственное решение $w$, для которого вследствие (2.84) справедливо неравенство

$$
\|w\|_{L_{q_{1} q_{2}}(D)} \leqslant c_{\eta} \varepsilon^{-2 / 3-8 \eta} .
$$

Возвращаясь к уравнению (2.59) и используя оценки разрешающего оператора $R_{\varepsilon}$, аналогично можно получить оценки $\partial^{\alpha} w,|\alpha|=0,1,2$.

ТЕОРема 2.2. Пусть $D$ - внешняя область с гладкой границей класса $C^{\infty}$. Тогда при достаточно малом $\varepsilon>0$ существует решение и задачи обтекания (0.1), бесконечно дифференцируемое в области $D$ вплоть до границы, причем для разности $u^{N}=u-e^{1}-v^{N}$ справедливы оценки

$$
\left\|u_{k}^{N}\right\|_{L_{q_{k}}(D)} \leqslant c_{\eta} \varepsilon^{N+1-2 / q_{k}-\eta}
$$

при $3<q_{1}<\infty, 2<q_{2}<\infty$,

$$
\left\|\partial^{\alpha} u^{N}\right\|_{L_{q}(D)} \leqslant c_{\eta} \varepsilon^{N+1-\eta},
$$

əде $3 / 2<q<\infty$ при $|\alpha|=1$ u $1<q<\infty$ nрu $|\alpha|=2$.

\section{Список литературы}

1. Ван-Дайк M. Методы возмущений в механике жидкости. М.: Мир, 1967.

2. Finn R., Smith D.R. On the stationary solutions of the Navier-Stokes equations in two dimensions // Arch. Rational Mech. Annal. 1967. V. 25. P. 26-39.

3. Срубщик Л. С., Юдович В. И. О применении метода Ньютона в задачах асимптотического интегрирования нелинейных уравнений // Тезисы Всесоюзной конференции по применению методов функционального анализа к решению нелинейных задач. Баку: Изд-во $\mathrm{AH} \mathrm{A} \mathrm{CCCP}, 1965$.

4. Белоносов C.M., Черноус K.A. Краевые задачи для уравнений Навье-Стокса. М.: Наука, 1985.

5. Трибель $X$. Теория интерполяции, функциональные пространства, дифференциальные операторы. М.: Мир, 1980.

6. Михлин С. Г. О мультипликаторах интегралов Фурье // ДАН СССР. 1956. Т. 109. C. $701-703$.

7. Красносельский М. А., Забрейко П. П., Пустыильник Е. И., Соболевский П. Е. Интегральные операторы в пространствах суммируемых функций. М.: Наука, 1966.

8. Лады женская О. А. Математические вопросы динамики вязкой несжимаемой жидкости. М.: ГИФМЛ, 1961.

9. Вайнберг M.M., Треногин В. А. Теория ветвления решений нелинейных уравнений. М.: Наука, 1969.

Ростовский государственный университет

г. Ростов-на-Дону

Поступило в редакцию

23.VII.2002 